\title{
T-dualization of Gödel string cosmologies via Poisson-Lie T-duality approach
}

\author{
Ali Eghbali ${ }^{\mathrm{a}}$, Reza Naderi ${ }^{\mathrm{b}}$, Adel Rezaei-Aghdam ${ }^{\mathrm{c}}$ \\ Department of Physics, Faculty of Basic Sciences, Azarbaijan Shahid Madani University, 53714-161, Tabriz, Iran
}

Received: 16 October 2020 / Accepted: 23 December 2020 / Published online: 21 January 2021

(C) The Author(s) 2021

\begin{abstract}
Using the homogeneous Gödel spacetimes we find some new solutions for the field equations of bosonic string effective action up to first order in $\alpha^{\prime}$ including both dilaton and axion fields. We then discuss in detail the (non)Abelian T-dualization of Gödel string cosmologies via the Poisson-Lie (PL) T-duality approach. In studying Abelian Tduality of the models we get seven dual models in such a way that they are constructed by one-, two- and three-dimensional Abelian Lie groups acting freely on the target space manifold. The results of our study show that the Abelian T-dual models are, under some of the special conditions, self-dual; moreover, by applying the usual rules of Abelian T-duality without further corrections, we are still able to obtain twoloop solutions. We also study the Abelian T-duality of Gödel string cosmologies up to $\alpha^{\prime}$-corrections by using the T-duality rules at two-loop order derived by Kaloper and Meissner. Afterwards, non-Abelian duals of the Gödel spacetimes are constructed by two- and three-dimensional non-Abelian Lie groups such as $A_{2}, A_{2} \oplus A_{1}$ and $\operatorname{SL}(2, \mathbb{R})$. In this way, the PL self-duality of $A d S_{3} \times \mathbb{R}$ space is discussed.
\end{abstract}

\section{Contents}

1 Introduction ................. . 2

2 Two-loop conformal invariance conditions of the bosonic string $\sigma$-model . . . . . . . . . . . . 3

3 Gödel spacetimes as solutions in string theory for the full $\mathcal{O}\left(\alpha^{\prime}\right)$ action . . . . . . . . . . . . . . . . 4

3.1 Gödel spacetimes in the cylindrical coordinates $(\hat{t}, \hat{r}, \hat{\varphi}, \hat{z}) \ldots \ldots \ldots 4 . \ldots \ldots$ 3.1.1 Solution up to zeroth order in $\alpha^{\prime}$. . . . . 4

3.1.2 Solutions up to first order in $\alpha^{\prime} \ldots$. . . . 4

3.2 Gödel spacetimes in new coordinates $(t, r, \varphi, z) \quad 5$

\footnotetext{
a e-mail: eghbali978@gmail.com (corresponding author)

be-mail: r_naderi8@yahoo.com

c e-mail: rezaei-a@azaruniv.ac.ir
}

3.2.1 Solution up to zeroth order in $\alpha^{\prime}$. . . . 5

3.2.2 Solutions up to first order in $\alpha^{\prime} \ldots$. . . . 5

4 A short review of PL T-duality with spectators . . . 6

5 Abelian T-dualization of Gödel string cosmologies . 8

5.1 Abelian T-duality with one-dimensional Abelian Lie group . . . . . . . . . . . . . . 8

5.1.1 Dualizing with respect to the coordinate $\varphi \quad 8$

5.1.2 Dualizing with respect to the coordinate $t \quad 9$

5.1.3 Dualizing with respect to the coordinate $z \quad 10$

5.2 Abelian T-duality with two-dimensional Abelian Lie group . . . . . . . . . . . . 10

5.2.1 Dualizing with respect to both coordinates $(t, \varphi) \ldots \ldots \ldots 10$

5.2.2 Dualizing with respect to both coordinates $(t, z) \ldots \ldots \ldots 11$

5.2.3 Dualizing with respect to both coordinates $(\varphi, z) \ldots \ldots \ldots 11$

5.3 Abelian T-duality with three-dimensional Abelian Lie group . . . . . . . . . . . . . . . 12

6 Abelian T-duality of Gödel string cosmologies up to $\alpha^{\prime}$-corrections . . . . . . . . . . . . 12

6.1 The $\alpha^{\prime}$-corrections from T-duality rules at twoloop .................. 12

6.1.1 Abelian T-duality with respect to the $z$ coordinate up to $\alpha^{\prime}$-corrections . . . . 16

6.1.2 Abelian T-duality with respect to the $t$ coordinate up to $\alpha^{\prime}$-corrections . . . . 17

7 Non-Abelian T-dualization of Gödel string cosmologies ................. . 19

7.1 Non-Abelian T-duality from a $2+2$-dimensional manifold with the $A_{2}$ Lie group . . . . . . . . . 19

7.2 Non-Abelian T-duality from a 3+1-dimensional manifold with the $A_{2} \oplus A_{1}$ Lie group . . . . . 20

7.3 Non-Abelian T-duality from a $3+1$-dimensional manifold with the $S L(2, \mathbb{R})$ Lie group . . . . . 22

8 Conclusion ................. 24

References................ 24 


\section{Introduction}

One of the most interesting cosmological solutions to Einstein's field equations is Gödel spacetime [1] which constituted (and still constitutes) a considerable motivation to the investigation of solutions more complex than those treated until then. It was obtained [2] some new solutions of string theory, including terms up to the first order in the inverse string tension $\alpha^{\prime}$ for the homogeneous Gödel spacetimes. Recently, it has been shown that [3] four-dimensional Gödel universe can be embedded in string theory. The corresponding Lagrangians to the Einstein-Maxwell-Axion, Einstein-Proca-Axion and Freedman-Schwarz $S U(2) \times$ $S U$ (2) gauged supergravity theories admit the Gödel metrics as solutions, all involving only the fundamental matter fields [3] (see also [4]). In Ref. [2], to find a class of Gödel universes without Closed Timelike Curves (CTC's) within the framework of low-energy effective string theory, it has been considered a convenient ansatz for both dilaton and axion fields in an orthonormal frame. Thus, the Gödel spacetimes are of particular importance both in general relativity and string theory. But so far their target space duality (T-duality) have not been studied. In the present work, we obtain the other forms of solutions of equations (but not in an orthonormal frame) for the two-loop beta-function including the Gödel spacetimes, field strength and dilaton field. The existence of solution for beta-function equations possessing the Gödel metrics with appropriate axion and dilaton fields, helps us to study their T-dual spaces. We furthermore get new solutions by considering a simpler form of Gödel metrics, and then focus on finding target space duals of the solutions. Accordingly, we improve Barrow's results [2].

T-duality is a very important symmetry of string theory which was originally defined for a string theory $\sigma$-model where the backgrounds of model have an Abelian group of isometries $[5,6]$. T-duality is a peculiar feature of strings, since it provides a method for relating seemingly inequivalent string theories, and allows to build new string backgrounds which could not be addressed otherwise. The theory of Abelian T-duality is well understood and had been the subject of much research (for a review see e.g. [7]). Then, the basic duality procedure could be generalized to the case where the original $\sigma$-model had a non-Abelian group of isometries [8] (further work in this direction was carried out in [9-14]). Whereas in the Abelian case, the dual theory has a natural symmetry suitable for inverting the duality transformation, in the non-Abelian case it has been shown that [11] this symmetry becomes nonlocal and cannot obviously be used for the inverse. In fact, it has been found that the non-Abelian T-duality is not an exact symmetry of the conformal field theory, and conjectured that it is a map between different conformal field theories. There has been a new interest in non-Abelian T-duality, which was ignited by [15], that provided the transformation rule for the Ramond-Ramond fields under the non-Abelian T-duality. It was then extended to $\sigma$-models with nonvanishing Ramond fluxes, thus allowing to search for new supergravity solutions [16-20]. Klimčik and Ševera proposed a generalization of T-duality, or the socalled PL T-duality $[21,22]$, which allows the duality to be performed on a target space without isometries. Afterwards, PL T-duality transformations could be generalized to the Lie supergroups [23], as well as supermanifolds [24], in such a way that super PL symmetry of the WZW models based on some of the Lie supergroups of superdimension (2|2) was studied $[25,26]$. Of course, in the Lie groups case, PL symmetry of the WZW models was already been studied in Ref. [27] (see also [28-30]). Recently, PL T-duality also appears as an important tool in the study of integrable models and their deformations [31-37]. Lately, using the PL T-duality approach in the presence of spectator fields we have found new dual solutions for some of the gravitational and string backgrounds such as BTZ black hole [38] and the WZW models based on the Lie groups $H_{4}$ and $G L(2, \mathbb{R})$ [39].

The main purpose of this paper is to study the (non)Abelian T-dualization (here as the PL T-duality on a (semi)Abelian double) of the Gödel string cosmologies. The procedure for this study is as follows: in the context of PL Tduality with spectators, the choice of spectator-dependent matrices $\left(E_{0}, F, F^{(1)}, F^{(2)}\right)$ plays a key role in the process. Our choice is usually based on the solutions of the vanishing of beta-function equations. By a suitable choice, we obtain the background of the original $\sigma$-model including the Gödel metrics and B-field (corresponding to a constant torsion). We then use the PL T-duality transformations in order to get the corresponding dual solutions. Also, the duality transformation of the dilaton field is, at one-loop level, given by equations (4.15) and (4.16). Finally, we are interested in testing the conformal invariance of the dual solutions up to the twoloop order. We notice that in the case of the Gödel spacetimes one can apply the usual rules of (non-)Abelian T-duality without further corrections, and still be able to obtain two-loop solutions. In general, further corrections to the rules are necessary. Recently, it has been shown that [40] (see also [41]) the PL duality can be extended to order $\alpha^{\prime}$, i.e. two loops in the $\sigma$-model perturbation theory, provided that the map is corrected. Furthermore, by using the higher derivative formulation of DFT, it has been found [42] a unified expression for first order corrections to generalized dualities so that it can be easily specified to any generalized T-duality (Abelian, non-Abelian, PL, etc.).

As explained above, we obtain the Abelian T-duals of the Gödel metrics by using the PL T-duality approach and then testing the conformal invariance conditions of the duals up to two-loop order. When going to two-loop it is known that in general the rules of Abelian T-duality must receive corrections. The two-loop $\sigma$-model corrections to the Abelian T- 
duality map were obtained by Kaloper and Meissner (KM) in [43]. They had used the effective action approach by focusing on backgrounds that have a single Abelian isometry. By using the T-duality rules of KM, we study the Abelian T-duality of Gödel string cosmologies up to $\alpha^{\prime}$-corrections when the dualizing is implemented by the shift of directions $z$ and $t$.

This paper is organized as follows. After the introduction section, Sect. 2 reviews the conformal invariance conditions of the $\sigma$-model up to the first order in $\alpha^{\prime}$. We start Sect. 3 by introducing the Gödel metrics and then discuss the solutions of two-loop beta-function equations possessing the Gödel spacetimes. A short review of PL T-dual $\sigma$-models construction in the presence of spectator fields is presented in Sect. 4, where necessary formulas are summarized. In Sect. 5, we study the Abelian T-dualization of the Gödel spacetimes via the PL T-duality approach. The study of the Abelian Tduality of the Gödel spacetimes up to $\alpha^{\prime}$-corrections using the $\mathrm{KM}$ approach, when the duality is implemented by a shift of the coordinates $z$ and $t$, is discussed in Sect. 6. The nonAbelian duals of the Gödel spacetimes constructed by twoand three-dimensional non-Abelian Lie groups are given in Sect. 7. In this section, we also study the PL self-duality of the $A d S_{3} \times \mathbb{R}$ space. The results of Abelian and non-Abelian T-duality of the Gödel string cosmologies are clearly summarized in Tables 1, 2 and 3. Some concluding remarks are given in the last section.

\section{Two-loop conformal invariance conditions of the bosonic string $\sigma$-model}

A bosonic string propagating on a non-trivial background can be described by the well-known $\sigma$-model defined on a twodimensional curved surface $\Sigma$ in $d$ spacetime dimensions with metric $G_{M N}$, antisymmetric tensor field $B_{M N}$ (axion field) and dilaton field $\Phi$

$$
\begin{aligned}
S= & \frac{1}{4 \pi \alpha^{\prime}} \int_{\Sigma} d \tau d \sigma \sqrt{-h}\left[h^{\alpha \beta} G_{M N}(X)+\epsilon^{\alpha \beta} B_{M N}(X)\right] \\
& \times \partial_{\alpha} X^{M} \partial_{\beta} X^{N}+\frac{1}{8 \pi} \int_{\Sigma} d \tau d \sigma R^{(2)} \Phi(X),
\end{aligned}
$$

where $\sigma^{\alpha}=(\tau, \sigma)$ are the string worldsheet coordinates, and $X^{M}(M=1, \ldots, d)$ are coordinates in spacetime. $h_{\alpha \beta}$ and $R^{(2)}$ are the induced metric and curvature scalar on the string worldsheet, respectively. $\epsilon^{\alpha \beta}$ is an antisymmetric tensor on the worldsheet and $h=\operatorname{det} h_{\alpha \beta}$. The dimensionful coupling constant $\alpha^{\prime}$ turns out to be the inverse string tension.

Since we are considering bosonic string theory, there is only one more massless degree of freedom of the string, namely the dilaton $\Phi$. This gives a contribution to the action in the form of the second term of (2.1). This term breaks Weyl invariance on a classical level as do the one-loop corrections to $G$ and $B$.

In the $\sigma$-model context, the conformal invariance conditions of the $\sigma$-model (2.1) are provided by the vanishing of the beta-function equations [44]. In order for the fields $(G, B, \Phi)$ to provide a consistent string background at low-energy up to two-loop order (first order in $\alpha^{\prime}$ ) they must satisfy the following equations [45-49]

$$
\begin{aligned}
& \mathcal{R}_{M N}-H_{M N}^{2}+\nabla_{M} \nabla_{N} \Phi+\frac{1}{2} \alpha^{\prime}\left[\mathcal{R}_{M P Q R} \mathcal{R}_{N}^{P Q R}+2 \mathcal{R}_{M P Q N} H^{2}{ }^{P Q}\right. \\
& +2 \mathcal{R}_{P Q R(M}{ }_{N}^{R S}{ }^{P Q}{ }_{S}^{P Q}+\frac{1}{3}\left(\nabla_{M} H_{P Q R}\right)\left(\nabla_{N} H^{P Q R}\right)-\left(\nabla_{P} H_{R S M}\right)\left(\nabla^{P} H_{N}^{R S}\right) \\
& \left.+2 H_{M P Q} H_{N R S} H^{T S Q} H_{T}^{R P}+2 H_{M P Q} H_{N R}^{Q} H^{2}{ }^{R P}\right]+\mathcal{O}\left(\alpha^{\prime 2}\right)=0 \\
& \nabla^{P} H_{P M N}-\left(\nabla^{P} \Phi^{\prime}\right) H_{M N P}+\alpha^{\prime}\left[\nabla^{P} H_{[M}^{R S} \mathcal{R}_{N] P R S}-\left(\nabla_{P} H_{R M N}\right) H^{2}{ }^{P R}\right. \\
& \left.-2\left(\nabla^{P}{ }^{Q R}{ }_{[M}\right) H_{N] Q S}{ }_{P R}^{S}\right]+\mathcal{O}\left(\alpha^{\prime 2}\right)=0, \\
& 2 \Lambda+\nabla^{2} \Phi^{\prime}-\left(\nabla \Phi^{\prime}\right)^{2}+\frac{2}{3} H^{2} \\
& -\alpha^{\prime}\left[\frac{1}{4} \mathcal{R}_{M N R S} \mathcal{R}^{M N R S}-\frac{1}{3}\left(\nabla_{M} H_{N R S}\right)\left(\nabla^{M} H^{N R S}\right)\right. \\
& -\frac{1}{2} H_{P}^{M N}{ }^{R S P} \mathcal{R}_{M N R S}-\mathcal{R}_{M N} H^{2}{ }^{M N}+\frac{3}{2} H_{M N}^{2} H^{2}{ }^{M N} \\
& \left.+\frac{5}{6} H_{M N P} H_{R S}^{M} H_{Q}^{N R} H^{P S Q}\right]+\mathcal{O}\left(\alpha^{\prime 2}\right)=0,
\end{aligned}
$$

where $H_{M N P}$ defined by $H_{M N P}=1 / 2\left(\partial_{M} B_{N P}+\partial_{N} B_{P M}+\right.$ $\left.\partial_{P} B_{M N}\right)$ is the field strength of the field $B_{M N}$. We have used the conventional notations $H_{M N}^{2}=H_{M P Q} H_{N}^{P Q}, H^{2}=$ $H_{M N P} H^{M N P}, H^{2^{M N}}=H^{M P Q} H_{P Q}{ }^{N}$ and $(\nabla \Phi)^{2}=\partial_{M} \Phi \partial^{M} \Phi$. $\mathcal{R}_{M N}$ and $\mathcal{R}_{M N P Q}$ are the Ricci tensor and Riemann tensor field of the metric $G_{M N}$, respectively. Moreover, in Eq. (2.4), $\Phi^{\prime}=\Phi+\alpha^{\prime} q H^{2}$ for some coefficient $q$ [48], and $\Lambda$ is a cosmological constant. In string theory, the $\Lambda$ is related to the dimension of spacetime, $d$, and the inverse string tension, $\alpha^{\prime}$, whereas in this paper it is, in some cases, treated as a free parameter. We note that round brackets denote the symmetric part on the indicated indices whereas square brackets denote the antisymmetric part.

On the other hand, the conditions for conformal invariance (Eqs. (2.2)-(2.4)) can be interpreted as field equations for $G_{M N}, B_{M N}$ and $\Phi$ of the string effective action [49]. As shown in Ref. [48], in $\mathrm{d}=26$ (where $\Lambda=0$ ), the string effective action up to the first order in $\alpha^{\prime}$ is given by

$$
\begin{aligned}
S_{\text {eff }}= & \int d^{D} X \sqrt{-G} e^{-\Phi}\left\{\mathcal{R}-\frac{1}{3} H^{2}+(\nabla \Phi)^{2}+\alpha^{\prime}\left[\frac{1}{4} \mathcal{R}_{M N P Q} \mathcal{R}^{M N P Q}\right.\right. \\
& -\frac{1}{2} \mathcal{R}_{M N P Q} H^{M N S} H_{S}^{P Q}+\frac{1}{6} H_{M N P} H^{P Q R} H_{R}{ }^{N S} H_{S Q}{ }^{M} \\
& +\frac{1}{2} H_{M N}^{2} H^{2^{M N}}-\mathcal{R}_{M N} \nabla^{M} \Phi \nabla^{N} \Phi \\
& -\frac{1}{6} H^{2}(\nabla \Phi)^{2}+\frac{1}{3} \nabla^{M} \Phi \nabla_{M} H^{2}-\frac{1}{6} \mathcal{R} H^{2}+\frac{1}{2} \mathcal{R}(\nabla \Phi)^{2}+\frac{1}{2}(\nabla \Phi)^{2} \nabla^{2} \Phi \\
& \left.\left.-\mathcal{R}_{M N} \mathcal{R}^{M N}+\frac{1}{36}\left(H^{2}\right)^{2}+\frac{1}{4} \mathcal{R}^{2}-\frac{1}{4}\left((\nabla \Phi)^{2}\right)^{2}\right]\right\}+\mathcal{O}\left(\alpha^{\prime 2}\right)
\end{aligned}
$$

As announced in the introduction, the Gödel spacetimes can be considered as exact solutions in string theory for the full 
$\mathcal{O}\left(\alpha^{\prime}\right)$ action including both dilaton and axion fields [2]. In Ref. [2], it has been considered a convenient ansatz for both dilaton and axion fields in an orthonormal frame. In the next section, we obtain the other forms of solutions for the twoloop beta-function equations including the Gödel spacetimes, the field strength $H$ and dilaton $\Phi$ in such a way that we do not work in an orthonormal frame.

\section{Gödel spacetimes as solutions in string theory for the full $\mathcal{O}\left(\alpha^{\prime}\right)$ action}

\subsection{Gödel spacetimes in the cylindrical coordinates $(\hat{t}, \hat{r}, \hat{\varphi}, \hat{z})$}

Among the known exact solutions of Einstein field equations, the Gödel and Gödel-type metrics [1] play a special role. It was shown within the usual general relativity that these solutions describe rotating string cosmologies, and allow for the existence of CTC's. It is a well-known result that all Gödeltype metrics, i.e., homogeneous spacetimes exhibiting vorticity, characterized by $\Omega$, and a given value of $m$ parameter can be rewritten in cylindrical coordinates $(\hat{t}, \hat{r}, \hat{\varphi}, \hat{z})$ as

$d s^{2}=-d \hat{t}^{2}-2 C(\hat{r}) d \hat{t} d \hat{\varphi}+G(\hat{r}) d \hat{\varphi}^{2}+d \hat{r}^{2}+d \hat{z}^{2}$,

where the functions $C(\hat{r})$ and $G(\hat{r})$ must obey the relations

$$
\begin{aligned}
& C(\hat{r})=\frac{4 \Omega}{m^{2}} \sinh ^{2}\left(\frac{m \hat{r}}{2}\right), \\
& G(\hat{r})=\frac{4}{m^{2}} \sinh ^{2}\left(\frac{m \hat{r}}{2}\right)\left[1+\left(1-\frac{4 \Omega^{2}}{m^{2}}\right) \sinh ^{2}\left(\frac{m \hat{r}}{2}\right)\right] .
\end{aligned}
$$

We note that $m^{2}=2 \Omega^{2}$ is a particular case of the hyperbolic class which corresponds to the original Gödel solution [1]. An interesting aspect of Gödel-type solutions is the possibility for existence of CTC's. The existence of CTC's, which allows for violation of causality, depends upon the sign of the metric function $G(\hat{r})$. Indeed, from Eqs. (3.1) and (3.2) one finds that the circles defined by $\hat{t}=t_{0}, \hat{r}=r_{0}, \hat{\varphi} \in[0,2 \pi], \hat{z}=z_{0}$ become CTC's whenever $G(\hat{r})<0$ [50]. In fact, the range, $m^{2} \geq 4 \Omega^{2}$, does not present CTC's.

\subsubsection{Solution up to zeroth order in $\alpha^{\prime}$}

The Eqs. (2.2)-(2.4) up to zeroth order in $\alpha^{\prime}$ are satisfied only when we have $m^{2}=4 \Omega^{2}$ with no CTC's. In this case, the field strength and dilaton field are, respectively, given by

$$
\begin{aligned}
H & =\frac{1}{2} \sinh (m \hat{r}) d \hat{t} \wedge d \hat{r} \wedge d \hat{\varphi}, \\
\Phi & =h \hat{z}+b,
\end{aligned}
$$

where $b$ is an arbitrary constant, and $h^{2}=2\left(\Lambda-2 \Omega^{2}\right)$. It is noteworthy that this solution is in agreement with those of Ref. [2].

\subsubsection{Solutions up to first order in $\alpha^{\prime}$}

Below we discuss the solutions of Eqs. (2.2)-(2.4) up to first order in $\alpha^{\prime}$ possessing the Gödel spacetimes in a coordinate base $(\hat{t}, \hat{r}, \hat{\varphi}, \hat{z})$. Our solutions are, in general, classified into two special classes:

Class I: In this class of solutions the field strength and dilaton field are, respectively, given by

$H=\mathbb{C} \sinh (m \hat{r}) d \hat{t} \wedge d \hat{r} \wedge d \hat{\varphi}, \quad \Phi=h \hat{z}+b$,

for some constants $\mathbb{C}, h, b$. The Eqs. (2.2)-(2.4) together with the fields given by Eq. (3.5) possess a Gödel solution with the metric (3.1) if the following conditions hold between the constants $m, \Omega, \mathbb{C}, h, \alpha^{\prime}$ and $\Lambda$ :

(i) The first constraint to satisfy the field equations (2.2) (2.4) with the metric (3.1) and the fields (3.5) is that

$$
\begin{gathered}
m^{2}=4 \Omega^{2}, \quad \alpha^{\prime}=\frac{1}{\Omega^{2}\left(1-12 \mathbb{C}^{2}\right)} \\
h^{2}=2 \Lambda-\Omega^{2} \frac{\left(176 \mathbb{C}^{4}-56 \mathbb{C}^{2}+3\right)}{\left(1-12 \mathbb{C}^{2}\right)} .
\end{gathered}
$$

This confirms that it is possible to obtain a Gödel solution with no CTC's. By noting the relation (3.6), the field strength $H$ depending on $\mathbb{C}$ can vanish. Then, the second relation of (3.6) gives the velocity of rotation of the Gödel universe in terms of the inverse string tension; moreover, there is another constraint, $h^{2}=2 \Lambda-3 \Omega^{2}$, which shows that the cosmological term has to be positive. This particular case, $\mathbb{C}=0$, is in agreement with those of Ref. [2].

(i') Similar to the case (i) we have $m^{2}=4 \Omega^{2}$ with no CTC's. In this case, the value of $\mathbb{C}$ is fixed to be $\mathbb{C}^{2}=1 / 4$, and $h^{2}=2 \Lambda-4 \Omega^{2}\left(1+2 \alpha^{\prime} \Omega^{2}\right)$. by

(ii) In this case, the relation between the constants is given

$\mathbb{C}^{2}=1-\frac{3 \Omega^{2}}{m^{2}}, \quad \alpha^{\prime}=\frac{1-\mathbb{C}^{2}}{\Omega^{2}\left(1-10 \mathbb{C}^{2}\right)}, \quad \frac{h^{2}}{2}=\Lambda-\Omega^{2} \frac{\left(1-4 \mathbb{C}^{2}\right)}{\left(1-\mathbb{C}^{2}\right)}$.

The first relation requires that $m^{2} \geq 3 \Omega^{2}$ for $\mathbb{C}^{2} \geq 0$. The range of $3 \Omega^{2} \leq m^{2}<4 \Omega^{2}$ allows CTC's. When $m^{2}=3 \Omega^{2}$, the field strength vanishes, then, $\alpha^{\prime}=1 / \Omega^{2}$ and $\Lambda>h^{2} / 2$.

(iii) The last case refers to the following relation between the constants $m, \Omega, \mathbb{C}, h, \alpha^{\prime}$, and $\Lambda$

$\mathbb{C}^{2}=\frac{\Omega^{2}}{m^{2}}, \quad \alpha^{\prime}=\frac{\mathbb{C}^{2}}{\Omega^{2}\left(1-6 \mathbb{C}^{2}\right)}, \quad h^{2}=2 \Lambda-\frac{\Omega^{2}\left(1-4 \mathbb{C}^{2}\right)^{2}}{\mathbb{C}^{2}\left(1-6 \mathbb{C}^{2}\right)}$

From the first relation of (3.8) one can easily deduce that the field strength must not vanish if a solution is to exist and act 
as a source of rotation. In this situation by fixing $\mathbb{C}^{2}$ to $1 / 2$ we then have the original Gödel solution ${ }^{1}$ for Eqs. (2.2)-(2.4).

Class II: The corresponding field strength to this class of solutions is given by

$H=\mathbb{C} \sinh (m \hat{r}) d \hat{t} \wedge d \hat{r} \wedge d \hat{\varphi}+\mathbb{D} \sinh (m \hat{r}) d \hat{r} \wedge d \hat{z} \wedge d \hat{\varphi}$,

for some constants $\mathbb{C}, \mathbb{D}$. In this case, dilaton field is assumed to be constant, $\Phi=b$. Using these, the Eqs. (2.2)-(2.4) with the metric (3.1) are satisfied if the following relation hold between the constants $m, \Omega, \mathbb{C}, \mathbb{D}, \alpha^{\prime}$ and $\Lambda$ :

$\mathbb{C}^{2}=\frac{216 \Omega^{4}-\left(108 m^{2}+48 \Lambda\right) \Omega^{2}+\left(17 m^{2}+6 \Lambda\right) m^{2}+\left(m^{2}-8 \Omega^{2}\right) \Gamma}{88 m^{2} \Omega^{2}}$,

$\alpha^{\prime}=\frac{\left(m^{2}-8 \Omega^{2}\right)}{2 m^{2} \Omega^{2}\left(11+6 \mathbb{C}^{2}\right)-3 m^{4}-44 \Omega^{4}}$,

$\mathbb{D}^{2}=\frac{\left(m^{2}-4 \Omega^{2}\right)\left[3 \Omega^{2}-m^{2}\left(1-\mathbb{C}^{2}\right)\right]}{m^{2}\left(m^{2}-8 \Omega^{2}\right)}$,

where

$\Gamma=\sqrt{80 \Omega^{4}-\left(104 m^{2}-160 \Lambda\right) \Omega^{2}+\left(25 m^{2}-60 \Lambda\right) m^{2}+36 \Lambda^{2}}$.

3.2 Gödel spacetimes in new coordinates $(t, r, \varphi, z)$

Since our main aim in the present work is to study the Tdualization of the Gödel spacetimes we write down the metric (3.1) in a simpler form. To this end, one uses the coordinate transformation

$$
\begin{aligned}
\sqrt{\beta} t & =\left\{\frac{\sqrt{\beta}}{l} \hat{t}-\hat{\varphi}+2 \arctan \left[e^{-m \hat{r}} \tan \left(\frac{\hat{\varphi}}{2}\right)\right]\right\}, \\
r & =\cosh (m \hat{r})+\cos \hat{\varphi} \sinh (m \hat{r}), \\
\sqrt{\beta} r \varphi & =\sin \hat{\varphi} \sinh (m \hat{r}), \\
z & =\frac{\hat{z}}{l},
\end{aligned}
$$

to obtain

$d s^{2}=l^{2}\left[-d t^{2}+\frac{d r^{2}}{r^{2}}+(\beta-1) r^{2} d \varphi^{2}-2 r d t d \varphi+d z^{2}\right]$,

such that the following relations must be held between the constants $l, \beta, m$ and $\Omega$ :

$l=\frac{1}{m}, \quad \beta=\frac{m^{2}}{4 \Omega^{2}}$

Here, the condition of the existence of CTC's, $m^{2}<4 \Omega^{2}$, is induced on the range of the parameter $\beta$. In fact, for the range $\beta \geq 1$ we do not encounter CTC's. In this case, the original Gödel metric [1] is recovered when we take $\beta=1 / 2$. As it is seen, the metric (3.12) is a direct product of $\mathbb{R}$ associated with the coordinate $z$ and the three-dimensional metric of

\footnotetext{
${ }^{1}$ In this case, one gets $\alpha^{\prime}=-\frac{1}{4 \Omega^{2}}$. In string theory, the resulting spectrum of the bosonic string contains a finite number of massless and infinitely many massive excitations with mass $^{2}=\frac{n}{\alpha^{\prime}}$ with $n \in N$. Among the states there are also tachyons with mass $^{2}<0$ implying that the vacuum is unstable. This is unavoidable in the bosonic string.
}

$(t, \varphi, r)$. For the case of $\beta=1$ one may use the following transformation

$d t=\rho(d \tau-d x), \quad r=\rho, \quad \varphi=x$,

then, the metric becomes

$d s^{2}=l^{2}\left[-\rho^{2} d \tau^{2}+\frac{d \rho^{2}}{\rho^{2}}+\rho^{2} d x^{2}+d z^{2}\right]$,

so when $\beta=1$, the metric (3.12) is locally $A d S_{3} \times \mathbb{R}$ space.

\subsubsection{Solution up to zeroth order in $\alpha^{\prime}$}

One can show that only $\beta=1$ case of the metric (3.12) satisfies the field equations (2.2)-(2.4) up to zeroth order in $\alpha^{\prime}$. In this case, a solution including the metric, field strength and dilaton field is given by

$$
\begin{aligned}
d s^{2} & =l^{2}\left[-d t^{2}+\frac{d r^{2}}{r^{2}}-2 r d t d \varphi+d z^{2}\right], \\
H & =\frac{l^{2}}{2} d t \wedge d r \wedge d \varphi \\
\Phi & =f z+b
\end{aligned}
$$

where $f^{2}=-1+2 l^{2} \Lambda$.

\subsubsection{Solutions up to first order in $\alpha^{\prime}$}

In order to obtain new solutions up to first order in $\alpha^{\prime}$ we now solve the field equations (2.2)-(2.4) for the metric (3.12). Here, the forms of our solutions including the field strength and dilaton field are given by two special classes A and B:

Class A: In this class of solutions the field strength $H$ and dilaton field $\Phi$ are given by

$H=\mathbb{E} d t \wedge d r \wedge d \varphi, \quad \Phi=f z+b$,

for some constants $\mathbb{E}, f, b$. The field equations (2.2)-(2.4) are then satisfied with the metric (3.12) together with the fields (3.17) if the following four conditions held between the constants $l, \beta, \mathbb{E}, f, \alpha^{\prime}$ and $\Lambda$ :

(1) The first condition is devoted to a special condition on the parameter $\beta$, and that is $\beta=1$. The rest of constants are then related to each other in the following way:

$$
\begin{aligned}
\alpha^{\prime} & =\frac{4 l^{6}}{l^{4}-12 \mathbb{E}^{2}}, \\
f^{2} & =\frac{176 \mathbb{E}^{4}+8 l^{4}\left(12 l^{2} \Lambda-7\right) \mathbb{E}^{2}+l^{8}\left(3-8 l^{2} \Lambda\right)}{4 l^{4}\left(12 \mathbb{E}^{2}-l^{4}\right)} .
\end{aligned}
$$

This result confirms that it is possible to have a solution corresponding to zero field strength, that is, $\mathbb{E}=0$. Then, it is followed that $\alpha^{\prime}=4 l^{2}$ and $f^{2}=2 l^{2} \Lambda-3 / 4$.

$\left(\mathbf{1}^{\prime}\right)$ In addition to the first condition, we have another solution corresponding to $\beta=1$ and nonzero field strength 
$\mathbb{E}^{2}=l^{4} / 4$, so that the relation between other constants may be expressed as $f^{2}=-1+2 l^{2} \Lambda-\alpha^{\prime} /\left(2 l^{2}\right)$.

(2) The field equations (2.2) and (2.3) are also fulfilled if the values of $\mathbb{E}$ and $\alpha^{\prime}$ can now be expressed in terms of the parameter $\beta$

$\mathbb{E}^{2}=\frac{l^{4}}{4}(4 \beta-3), \quad \alpha^{\prime}=\frac{2 \beta l^{2}}{5-6 \beta}$.

This is an interesting case in which the field strength depends on the parameter $\beta$ of the metric. From the relation (3.19) one can easily deduce that $\beta \in[3 / 4,+\infty)-\{5 / 6\}$. We note that in this case, the range of $\beta \in[3 / 4,1)-\{5 / 6\}$ allows CTC's. The relation between other constants can be obtained from Eq. (2.4), which gives, $f^{2}=2\left(1+l^{2} \Lambda-1 / \beta\right)$.

(3) The last condition refers to a Gödel solution with a nonzero field strength, $\mathbb{E}^{2}=l^{4} / 4$. From Eqs. (2.2) and (2.3) the value of $\alpha^{\prime}$ is expressed in terms of the parameter $\beta$, obtaining

$\alpha^{\prime}=\frac{2 \beta l^{2}}{2 \beta-3}$.

Finally after using Eq. (2.4), we obtain the relation

$\frac{f^{2}}{2}=\frac{\beta^{2}\left(2 l^{2} \Lambda-1\right)+\beta\left(2-3 l^{2} \Lambda\right)-1}{\beta(2 \beta-3)}$.

This is only case of the class A which is valid for $\beta=1 / 2$. Putting $\beta=1 / 2$, we then get $\alpha^{\prime}=-l^{2} / 2$. Also, from Eq. (3.21) it is followed that $\Lambda \geq-1 /\left(4 l^{2}\right)$, which shows that the cosmological term can be considered to be negative.

Class B: Class B of solutions is devoted to a constant dilaton field $\Phi=b$ and the field strength

$H=\mathbb{E} d t \wedge d r \wedge d \varphi+\mathbb{N} d r \wedge d \varphi \wedge d z$

for some constants $\mathbb{E}, \mathbb{N}$, together with the metric (3.12). The equations of motion (2.2)-(2.4) are then fulfilled in this general case. From Eqs. (2.2) and (2.3) we obtain

$$
\begin{aligned}
\alpha^{\prime} & =\frac{4 \beta l^{6}(\beta-2)}{l^{4}\left(12 \beta^{2}-22 \beta+11\right)-12 \mathbb{E}^{2}}, \\
\mathbb{N}^{2} & =\frac{(\beta-1)\left[4 \mathbb{E}^{2}+l^{4}(3-4 \beta)\right]}{4(\beta-2)} .
\end{aligned}
$$

Finally, Eq. (2.4) is satisfied if

$\mathbb{E}^{2}=\frac{l^{4}}{44}\left[34 \beta^{2}-54 \beta+12 \beta l^{2} \Lambda(\beta-2)+27+2(\beta-2) \Xi\right]$,

where $\Xi=\sqrt{\beta^{2}\left(5-6 l^{2} \Lambda\right)^{2}+2 \beta\left(20 l^{2} \Lambda-13\right)+5}$. We have thus obtained some new solutions for the field equations of bosonic string effective action up to the first order in $\alpha^{\prime}$ in the forms of classes A and B. These solutions will be useful in studying the T-dualization of Gödel string cosmologies. We shall address this problem in Sects. 5, 6 and 7.

\section{A short review of PL T-duality with spectators}

In this section, we recall the main features of PL T-duality transformations at the level of the $\sigma$-model. For the description of PL T-duality, we need to introduce the Drinfeld double group $D$ [51], which by definition has a pair of maximally isotropic subgroups $G$ and $\tilde{G}$ corresponding to the subalgebras $\mathcal{G}$ and $\tilde{\mathcal{G}}$, respectively. The generators of $\mathcal{G}$ and $\tilde{\mathcal{G}}$ are denoted, respectively, $T_{a}$ and $\tilde{T}^{a}, a=1, \ldots, \operatorname{dim} G$ and satisfy the commutation relations

$$
\begin{aligned}
& {\left[T_{a}, T_{b}\right]=f_{a b}^{c} T_{c}, \quad\left[\tilde{T}^{a}, \tilde{T}^{b}\right]=\tilde{f}_{c}^{a b} \tilde{T}^{c},} \\
& {\left[T_{a}, \tilde{T}^{b}\right]=\tilde{f}_{a}^{b c} T_{c}+f_{c a}^{b} \tilde{T}^{c} .}
\end{aligned}
$$

The Lie algebra structure defined by (4.1) is called the Drinfeld double $\mathcal{D}$. The structure constants $f^{c}{ }_{a b}$ and $\tilde{f}_{c}^{a b}$ are subject to the Jacobi identities and the following mixed Jacobi identities

$$
f_{b c}^{a} \tilde{f}_{a}^{d e}=f^{d}{ }_{a c} \tilde{f}^{a e}{ }_{b}+f^{e}{ }_{b a} \tilde{f}^{d a}{ }_{c}+f^{d}{ }_{b a} \tilde{f}^{a e}{ }_{c}+f^{e}{ }_{a c} \tilde{f}^{d a}{ }_{b} .
$$

In addition, the Drinfeld double $\mathcal{D}$ is equipped with an invariant inner product $\langle.,$.$\rangle with the following properties$

$$
\begin{aligned}
& \left\langle T_{a}, \tilde{T}^{b}\right\rangle=\delta_{a}{ }^{b}, \\
& \left\langle T_{a}, T_{b}\right\rangle=\left\langle\tilde{T}^{a}, \tilde{T}^{b}\right\rangle=0 .
\end{aligned}
$$

Let us now consider a $d$-dimensional manifold $\mathcal{M}$ and some coordinates $X^{M}=\left(x^{\mu}, y^{i}\right)$ on it, where $x^{\mu}, \mu=$ $1, \ldots, \operatorname{dim} G$ stand for the coordinates of Lie group $G$ acting freely from right on $\mathcal{M}$ and $y^{i}, i=1, \ldots, d-\operatorname{dim} G$ are the coordinates labeling the orbit $O$ of $G$ in the target space $\mathcal{M}$. We note that the coordinates $y^{i}$ do not participate in the PL T-duality transformations and are therefore called spectators [28].

Before proceeding to write the $\sigma$-models let us introduce the components of the right-invariant Maurer-Cartan forms $\left(g^{-1} \partial_{\alpha} g\right)^{a} \equiv R_{\alpha}^{a}=\partial_{\alpha} x^{\mu} R_{\mu}^{a}$, where $g$ is an element of the Lie group $G$ corresponding to the Lie algebra $\mathcal{G}$. For notational convenience we will also use $R_{\alpha}^{i}=\partial_{\alpha} y^{i}$. In order to define $\sigma$-models with PL T-duality symmetry, it is convenient to define matrices $a(g), b(g)$ and $\Pi(g)$ in the following way

$$
\begin{aligned}
g^{-1} T_{a} g & =a_{a}^{b}(g) T_{b}, \\
g^{-1} \tilde{T}^{a} g & =b^{a b}(g) T_{b}+\left(a^{-1}\right)_{b}^{a}(g) \tilde{T}^{b}, \\
\Pi^{a b}(g) & =b^{a c}(g)\left(a^{-1}\right)_{c}^{b}(g) .
\end{aligned}
$$

Then, the original $\sigma$-model is defined by the action $[21,22$, 28]

$$
\begin{aligned}
S= & \frac{1}{2} \int d \sigma^{+} d \sigma^{-}\left[E_{a b} R_{+}^{a} R_{-}^{b}+\phi_{a j}^{(1)} R_{+}^{a} \partial_{-} y^{j}+\phi_{i b}^{(2)} \partial_{+} y^{i} R_{-}^{b}+\phi_{i j} \partial_{+} y^{i} \partial_{-} y^{j}\right] \\
& -\frac{1}{4 \pi} \int d \sigma^{+} d \sigma^{-} R^{(2)} \Phi(X) .
\end{aligned}
$$

Here, we have used the standard light-cone variables on the worldsheet, $\sigma^{ \pm}=(\tau \pm \sigma) / 2$ together with $\partial_{ \pm}=\partial_{\tau} \pm \partial_{\sigma}$. 
The backgrounds appearing in this action are given in matrix notation by [28]

$$
\begin{aligned}
E & =\left(E_{0}^{-1}+\Pi\right)^{-1}, \\
\phi^{(1)} & =E E_{0}^{-1} F^{(1)}, \\
\phi^{(2)} & =F^{(2)} E_{0}^{-1} E, \\
\phi & =F-F^{(2)} \Pi E E_{0}^{-1} F^{(1)} .
\end{aligned}
$$

The matrices $\left(E_{0}, F, F^{(1)}, F^{(2)}\right)$ are all functions of the variables $y^{i}$ only.

Similarly we consider another $\sigma$-model for the $d$ field variables $\tilde{X}^{M}=\left(\tilde{x}^{\mu}, y^{i}\right)$, where $\tilde{x}^{\mu}, \mu=1,2, \ldots, \operatorname{dim} \tilde{G}$ parameterize an element $\tilde{g}$ of a Lie group $\tilde{G}$, whose dimension is, however, equal to that of $G$. The rest of the variables are the same $y^{i}$ used in (4.5). Accordingly, we introduce the components of the right-invariant Maurer-Cartan forms $\left(\tilde{g}^{-1} \partial_{ \pm} \tilde{g}\right)_{a}=\tilde{R}_{ \pm_{a}}=\partial_{ \pm} \tilde{x}^{\mu} \tilde{R}_{\mu a}$ on the Lie group $\tilde{G}$. The corresponding $\sigma$-model has the form

$$
\begin{aligned}
& \tilde{S}=\frac{1}{2} \int d \sigma^{+} d \sigma^{-}\left[\begin{array}{lll}
\tilde{E}^{a b} & \tilde{R}_{+_{a}} \tilde{R}_{-b} \\
&
\end{array}\right. \\
& \left.+\tilde{\phi}_{j}^{(1)^{a}} \tilde{R}_{+_{a}} \partial_{-} y^{j}+\tilde{\phi}_{i}^{(2)^{b}} \partial_{+} y^{i} \tilde{R}_{-b}+\tilde{\phi}_{i j} \partial_{+} y^{i} \partial_{-} y^{j}\right] \\
& -\frac{1}{4 \pi} \int d \sigma^{+} d \sigma^{-} R^{(2)} \tilde{\Phi}(\tilde{X}) .
\end{aligned}
$$

The backgrounds of the dual theory are related to those of the original one by $[21,28]$

$$
\begin{aligned}
& \tilde{E}=\left(E_{0}+\tilde{\Pi}\right)^{-1}, \\
& \tilde{\phi}^{(1)}=\tilde{E} F^{(1)}, \\
& \tilde{\phi}^{(2)}=-F^{(2)} \tilde{E}, \\
& \tilde{\phi}=F-F^{(2)} \tilde{E} F^{(1)},
\end{aligned}
$$

where $\tilde{\Pi}$ is defined as in (4.4) by replacing untilded quantities with tilded ones.

Notice that the duality transformation must be supplemented by a correction that comes from integrating out the fields on the dual group in path integral formulation in such a way that it can be absorbed at the one-loop level into the transformation of the dilaton field. The correct formula of dilaton transformation is, for the PL T-duality case, given by [52]

$\Phi=\phi^{(0)}+\log |\operatorname{det} E|-\log \left|\operatorname{det} E_{0}\right|-\log |\operatorname{det} a(g)|$,

$\tilde{\Phi}=\phi^{(0)}+\log |\operatorname{det} \tilde{E}|-\log |\operatorname{det} \tilde{a}(\tilde{g})|$,

where $\phi^{(0)}$ is the dilaton that makes the original $\sigma$-model conformal (up to the one-loop order) and may depend on both group and spectator coordinates. Accordingly, the dual background can also be conformal at the one-loop level with a new dilaton field obtaining from Eq. (4.16). The transformations (4.15) and (4.16) were based on a regularization of a functional determinant in the path integral formulation of PL duality [52] (see also [53]). PL T-duality is a canonical transformation and two $\sigma$-models related by PL duality are, therefore, equivalent at the classical level [54]. It has been shown that [55] relations (4.15) and (4.16) only hold at the one-loop level for both $\sigma$-models admitting PL duality if the traces of the structure constants of each Lie algebra constituting the Drinfeld double are zero. Equivalence can hold in some special cases but it fails in most cases. In this respect, checking the equivalence by studying conformal invariance (the vanishing of the beta-function equations) is important. But, since after a classical canonical transformation, the equivalence always holds up to first order in Planck's constant in the semiclassical expansion (corresponding to one-loop order in $\sigma$-model language), only the two-loop order is the first real test of quantum equivalence of the two different $\sigma$-models related by PL T-duality [40,41]. As mentioned in the introduction section, in the case of Abelian T-duality, the two-loop $\sigma$-model corrections were obtained by KM in [43]. There, one can find the duality transformation of the dilaton field at two-loop level.

Let us turn into the actions (4.5) and (4.10). These actions correspond to PL T-dual $\sigma$-models [21,22]. If the group $G(\tilde{G})$ besides having free action on the manifold $\mathcal{M}(\tilde{\mathcal{M}})$, acts transitively on it, then the corresponding manifold $\mathcal{M}(\tilde{\mathcal{M}})$ will be the same as the group $G(\tilde{G})$. In this case only the first term appears in the actions (4.5) and (4.10). The T-duality transformations are said to be Abelian $[5,6]$ or non-Abelian $[8,12]$ according to the nature of the Lie algebra formed by the generators of the isometries. Notice that:

- If both the Lie groups $G$ and $\tilde{G}$ become the isometry groups of the manifolds $\mathcal{M}$ and $\tilde{\mathcal{M}}$, respectively, namely, both $G$ and $\tilde{G}$ are chosen to be Abelian groups $\left(f_{b c}^{a}=\right.$ $\left.\tilde{f}_{c}^{a b}=0\right)$, then we get $\Pi(g)=\tilde{\Pi}(\tilde{g})=0$, recovering thus the standard Abelian duality. In this case, the dilaton transformation (4.15) turns into $\Phi=\phi^{(0)}$. By remembering that $\phi^{(0)}$ makes the original model conformal at one-loop level we obtain the final result for the dual dilaton, giving

$\tilde{\Phi}=\phi^{(0)}-\log \left|\operatorname{det} E_{0}\right|$.

Indeed, this new dilaton field guaranties the conformality of dual background up to the one-loop order.

- In case of non-Abelian T-duality the former represents group of symmetries of the original $\sigma$-model, while the latter is Abelian $\left(\tilde{f}_{c}^{a b}=0\right)$. Furthermore, there are Drinfeld doubles where both $G$ and $\tilde{G}$ are non-Abelian. In such a case the symmetry of the original model is replaced with the so-called PL symmetry (or generalized symmetry), and the full PL Tduality transformation $[21,22]$ applies. We now wish to apply 
the above discussions to study the Abelian T-dualization of the Gödel string cosmologies in the next section.

\section{Abelian T-dualization of Gödel string cosmologies}

In order to study Abelian T-duality in Buscher's construction [5,6] one starts with a manifold $\mathcal{M}$ with metric $G_{M N}$, antisymmetric tensor $B_{M N}$ and dilaton field $\Phi$, and requires the metric to admit at least one continuous Abelian isometry leaving invariant the action of $\sigma$-model constructed out of $(G, B, \Phi)$. As announced in the introduction, PL Tduality proposed by Klimčik and Ševera is a generalization of Abelian and non-Abelian T-dualities. It was then shown that Buscher's duality transformations can be obtained from the PL T-duality approach by a convenient choice of the spectator-dependent matrices [56] (see also [38]). In this section, we obtain all possible Abelian T-duals of the Gödel string cosmologies by using the approach of PL Tduality in the presence of spectators. In this regard, the Lie groups $G$ and $\tilde{G}$ acting freely on the target space manifolds $\mathcal{M} \approx O \times G$ and $\tilde{\mathcal{M}} \approx O \times \tilde{G}$, respectively, can be considered to be Abelian Lie groups of dimensions one, two and three. In all cases, both structures $\Pi(g)$ and $\tilde{\Pi}(\tilde{g})$ are vanished as mentioned above.

When the dualizing is implemented by the shift of the directions $t, z$ and $(t, z)$ we show that the pair of the mutually dual models can be conformally invariant at the one-loop level, in a way that the corresponding dual dilaton field is found by using transformation (4.17). It is shown that for the duals obtained through the dualizing on these directions without further corrections, we are still able to obtain twoloop solutions. On the other hand, the dual models obtained by the rest of the directions, $\varphi,(t, \varphi),(\varphi, z)$ and $(t, \varphi, z)$, don't remain conformally invariant at the one-loop level; we just check their two-loop conformal invariance conditions.

\subsection{Abelian T-duality with one-dimensional Abelian Lie group}

\subsubsection{Dualizing with respect to the coordinate $\varphi$}

Here we assume that target space $\mathcal{M} \approx O \times G$ is defined by coordinates $X^{M}=(x ; r, t, z)$. The coordinates of orbit $O$ are represented by $y^{i}=(r, t, z)$, while $x$ is the coordinate of one-dimensional Abelian Lie group $G=A_{1}$ parameterizing by element $g=e^{x T}$. In order to write the original $\sigma$ model on the manifold $\mathcal{M}$ we need to determine the model couplings. Let us choose the spectator-dependent matrices in the following form

$$
E_{0} a b=\mathcal{C}_{0} r^{2}, \quad F_{a j}^{(1)}=\left(\begin{array}{lll}
F_{1 \overline{1}}^{(1)} & F_{1 \overline{2}}^{(1)} & f_{3}(r, t, z)
\end{array}\right),
$$

$$
\begin{aligned}
F_{i j} & =\left(\begin{array}{ccc}
\frac{l^{2}}{r^{2}} & f_{1}(r, t, z) & f_{2}(r, t, z) \\
-f_{1}(r, t, z) & -l^{2} & F_{23} \\
-f_{2}(r, t, z) & -F_{23} & l^{2}
\end{array}\right), \\
F_{i b}^{(2)} & =-\left(\begin{array}{c}
F_{1 \overline{1}}^{(1)} \\
F_{1 \overline{2}}^{(1)}+2 \gamma_{0} l^{2} r \\
f_{3}(r, t, z)
\end{array}\right) .
\end{aligned}
$$

where $\mathcal{C}_{0}$ is a nonzero constant and

$$
\begin{aligned}
\gamma_{0}^{2}= & \frac{\mathcal{C}_{0}}{l^{2}(\beta-1)} \\
F_{1 \overline{1}}^{(1)}= & h_{1}(r)+\int\left[\partial_{r} f_{3}(r, t, z)-2 \mathbb{N} \gamma_{0}\right] d z \\
& +\int\left[l^{2} \gamma_{0}\left(1-\frac{2 \mathbb{E}}{l^{2}}\right)+\partial_{r} h_{2}(r, t)\right] d t, \\
F_{1 \overline{2}}^{(1)}= & h_{2}(r, t)+\int \partial_{t} f_{3}(r, t, z) d z, \\
F_{23}= & h_{3}(t, z)+\int\left[\partial_{t} f_{2}(r, t, z)-\partial_{z} f_{1}(r, t, z)\right] d r,
\end{aligned}
$$

in which $h_{i}$ 's and $f_{i}$ 's are some arbitrary functions that may depend on some coordinates $r, t$ and $z$. Henceforth, the constants $\mathbb{E}, \mathbb{N}, l$ and $\beta$ are the same ones introduced in Sect. 3 . Inserting (5.1) into Eqs. (4.6)-(4.9) and then employing (4.5), the original $\sigma$-model on the manifold $\mathcal{M}$ can be derived. Then corresponding metric and antisymmetric tensor may be expressed as

$$
\begin{aligned}
d s^{2}= & l^{2}\left[-d t^{2}+\frac{d r^{2}}{r^{2}}+(\beta-1) \gamma_{0}^{2} r^{2} d x^{2}-2 r \gamma_{0} d t d x+d z^{2}\right] \\
B= & f_{1}(r, t, z) d r \wedge d t+f_{2}(r, t, z) d r \wedge d z \\
& +F_{23} d t \wedge d z+f_{3}(r, t, z) d x \wedge d z \\
& \left.+F_{1 \overline{1}}^{(1)} d x \wedge d r+\left(\gamma_{0} l^{2} r+F_{1 \overline{2}}^{(1)}\right) d x \wedge d t\right]
\end{aligned}
$$

Carrying out the coordinate transformation $\varphi=\gamma_{0} x$ we see that (5.3) is nothing but the Gödel metric (3.12). Furthermore, one concludes that the field strength corresponding to the $B$-field (5.4) is the same as (3.22). We note the fact that in the context of PL T-duality, the matrix $E_{0}$ must be invertible. With regard to the first relation of (5.2) the solution given by (5.3) and (5.4) is valid for all values of $\beta$ except for 1 . Therefore, the original model don't remain conformally invariant at one-loop level as mentioned in Sect. 3. Finally, one can just verify the field equations (2.2)-(2.4) for the metric (5.3) and the $B$-field (5.4) with a constant dilaton field.

The dual background is obtained from a $\sigma$-model which is constructed on $1+3$-dimensional manifold $\tilde{\mathcal{M}} \approx O \times \tilde{G}$ where $\tilde{G}$ is the same as the $A_{1}$ Lie group. Since duality is performed on the $x$, we parameterize $\tilde{G}$ by the coordinate $\tilde{x}$. Before proceeding to construct the dual background, let us consider a simpler form of the spectator-dependent matrices (5.1). Indeed, one may take $h_{1}=h_{3}=f_{1}=f_{2}=0, h_{2}=$ 
$\left(2 \mathbb{E}-l^{2}\right) r, f_{3}=2 \mathbb{N} r$ and $\mathcal{C}_{0}=l^{2}(\beta-1)$. Imposing these conditions on (5.1) and then utilizing Eqs. (4.11)-(4.14) and also (4.10), the metric and antisymmetric tensor field $\tilde{B}$ of the dual model can be cast in the forms

$$
\begin{aligned}
d \tilde{s}^{2}= & \frac{l^{2} d r^{2}}{r^{2}}+\frac{1}{(\beta-1)}\left[\left(\frac{4 \mathbb{E}^{2}}{l^{2}}-\beta l^{2}\right) d t^{2}\right. \\
& +\frac{d \tilde{x}^{2}}{l^{2} r^{2}}+\left(\frac{4 \mathbb{N}^{2}}{l^{2}}+l^{2}(\beta-1)\right) d z^{2} \\
& +\frac{8 \mathbb{E} \mathbb{N}}{l^{2}} d t d z \\
& \left.+\frac{4 \mathbb{E}}{l^{2} r} d \tilde{x} d t+\frac{4 \mathbb{N}}{l^{2} r} d \tilde{x} d z\right], \\
\tilde{B}= & \frac{1}{(\beta-1)}\left(\frac{1}{r} d t \wedge d \tilde{x}+2 \mathbb{N} d t \wedge d z\right) .
\end{aligned}
$$

One quickly deduces that the only nonzero component of the field strength corresponding to the $\tilde{B}$-field (5.6) is

$\tilde{H}_{\tilde{x} t r}=\frac{1}{2 r^{2}(\beta-1)}$.

Let us now clarify the spacetime structure and conformal invariance conditions of the dual model. We see that the dual background can't, for the $\beta \neq 1$ case, satisfy the field equations (2.2)-(2.4) up to zeroth order in $\alpha^{\prime}$ (oneloop order). Accordingly, the dual dilaton don't obey transformation (4.17). Below, we just check the two-loop conformal invariance conditions of the dual background. When $\mathbb{N}=0$, the metric (5.5) and the field strength (5.7) satisfy the field equations (2.2)-(2.4) with a dilaton field in the form of $\tilde{\Phi}=\tilde{f} z+b$ provided that the constants $\mathbb{E}, l, \tilde{f}, \alpha^{\prime}$ and $\beta$ satisfy the following relations

$\mathbb{E}^{2}=\frac{l^{4}}{4}, \quad \alpha^{\prime}=\frac{2 l^{2} \beta}{2 \beta-3}$,

$\tilde{f}^{2}=\frac{2 \beta^{2}\left(2 \tilde{\Lambda} l^{2}-1\right)-2 \beta\left(3 \tilde{\Lambda} l^{2}-2\right)-2}{\beta(2 \beta-3)}$.

Imposing conditions $\mathbb{N}=0$ and $\mathbb{E}^{2}=l^{4} / 4$ on the metric (5.5) and then performing the coordinate transformation $r \rightarrow$ $1 / r, \tilde{x} \rightarrow-l^{2}(\beta-1) \varphi$ one concludes that it is nothing but the Gödel metric (3.12). ${ }^{2}$ This means that the model is selfdual. Also when $\mathbb{N}=0$ we have another solution in which relationship between the constants are given by

$\mathbb{E}^{2}=\frac{l^{4}(4 \beta-1)}{12}, \quad \alpha^{\prime}=\frac{6 l^{2} \beta}{2 \beta-5}$,

$\tilde{f}^{2}=\frac{2}{3 \beta}\left(1+\beta\left(3 \tilde{\Lambda} l^{2}-1\right)\right)$.

In addition to the above, for the metric (5.5) and the field strength (5.7) one verifies the field equations (2.2)-(2.4) with

2 We recall that in the Abelian case with $U(1)$ duality group and another $U(1)$ co-duality group, the target space is a one-dimensional circle; in other words, we have here the standard $R \rightarrow 1 / R$ duality $[5,6]$. a constant dilaton field when $\mathbb{N}$ differs from zero. In this way we must have

$$
\begin{aligned}
\mathbb{E}^{2} & =\frac{l^{4}}{4}\left(2 \beta^{2}-2 \beta+1\right), \quad \mathbb{N}^{2}=\frac{l^{4}}{2}(\beta-1)^{2}, \quad \alpha^{\prime}=\frac{2 l^{2} \beta}{2-3 \beta}, \\
\tilde{\Lambda} & =\frac{(\beta-1)^{2}}{4 l^{2} \beta(2-3 \beta)} .
\end{aligned}
$$

\subsubsection{Dualizing with respect to the coordinate $t$}

In this case the coordinates of the target manifold $\mathcal{M}$ are denoted by $(t ; r, \varphi, z)$ wherein $t$ is the coordinate of the Lie group $G$ which the duality is performed on, while $(r, \varphi, z)$ are the coordinate representations of the orbit $O$. If we choose the coupling matrices as

$$
\begin{aligned}
F_{a j}^{(1)} & =\left(\begin{array}{lll}
0 & -r\left(2 \mathbb{E}+l^{2}\right) & 0
\end{array}\right), F_{i b}^{(2)}=\left(\begin{array}{c}
0 \\
r\left(2 \mathbb{E}-l^{2}\right) \\
0
\end{array}\right), \\
F_{i j} & =\left(\begin{array}{ccc}
\frac{l^{2}}{r^{2}} & 0 & 0 \\
0 & (\beta-1) l^{2} r^{2} & 2 \mathbb{N} r \\
0 & -2 \mathbb{N} r & l^{2}
\end{array}\right),
\end{aligned}
$$

and $E_{0 a b}=-l^{2}$, then using the formulae (4.6)-(4.9) and also (4.5) one finds that the metric and field strength corresponding to the original $\sigma$-model take the same form as (3.12) and (3.22), respectively. According to the results of Sect. 3, the $\beta=1$ case of the Gödel spacetimes with $\mathbb{E}^{2}=l^{4} / 4$ and $\mathbb{N}=0$ satisfy the one-loop beta-function equations. In this regard, the dilaton field that makes the original $\sigma$-model conformal is found to be $\Phi=\phi^{(0)}$. However, according to Sect. 3 since we want the total dilaton to be $\Phi=f z+b$ we need to choose $\phi^{(0)}=f z+b$ in which $f^{2}=-1+2 l^{2} \Lambda$.

It is then straightforward to compute the corresponding dual spacetime. They are read

$$
\begin{aligned}
d \tilde{s}^{2} & =\frac{l^{2} d r^{2}}{r^{2}}+l^{2} d z^{2}-\frac{1}{l^{2}} d \tilde{x}^{2}+\left(\beta l^{2}-\frac{4 \mathbb{E}^{2}}{l^{2}}\right) r^{2} d \varphi^{2}+\frac{4 \mathbb{E}}{l^{2}} r d \tilde{x} d \varphi, \\
\tilde{H} & =-\frac{1}{2} d r \wedge d \varphi \wedge d \tilde{x}+\mathbb{N} d r \wedge d \varphi \wedge d z,
\end{aligned}
$$

where $\tilde{x}$ is the dualized coordinate of the dual manifold. We note that the background (5.12), unlike the dual background in the preceding case, can be even conformally invariant up to the zeroth order in $\alpha^{\prime}$. For the $\beta=1$ case of this background the dual dilaton is found by using Eq. (4.17) to be $\tilde{\Phi}=$ $\phi^{(0)}-\log l^{2}$. Finally we get the dilaton by remembering that $\phi^{(0)}=f z+b$ which gives the final result

$\tilde{\Phi}=f z+b-\log l^{2}$.

In addition, the case of $\beta=1$ can satisfy the field equations (2.2)-(2.4) with the same dilaton field (5.13) provided that

$\mathbb{E}^{2}=\frac{l^{4}}{4}, \quad \mathbb{N}=0, \quad f^{2}=-\frac{\alpha^{\prime}}{2 l^{2}}+2 l^{2} \Lambda-1$,

for each $\alpha^{\prime}$. Actually, by imposing the conditions $2 \mathbb{E}=-l^{2}$ and $\mathbb{N}=0$ on the dual background (5.12) and then by making 
use of the transformation $\tilde{x} \rightarrow l^{2} t$, we obtain the same forms as (3.12) and (3.17), that is, the model is self-dual.

We can also show that the dual background is conformally invariant up to first order in $\alpha^{\prime}$ when $\mathbb{N}$ differs from zero. In this way, if we consider

$$
\begin{aligned}
\mathbb{E}^{2} & =\frac{l^{4}}{4}, \quad \mathbb{N}^{2}=\frac{l^{4}(\beta-1)^{2}}{2-\beta}, \quad \alpha^{\prime}=\frac{2 l^{2} \beta(2-\beta)}{6 \beta^{2}-11 \beta+4}, \\
\tilde{\Lambda} & =\frac{1}{\beta l^{2}}\left(\frac{-6 \beta^{4}+18 \beta^{3}-18 \beta^{2}+7 \beta-1}{6 \beta^{3}-23 \beta^{2}+26 \beta-8}\right) .
\end{aligned}
$$

then the field equations (2.2)-(2.4) for (5.12) are satisfied with a constant dilaton.

\subsubsection{Dualizing with respect to the coordinate $z$}

In what follows we shall dualize the Gödel metrics on the coordinate $z$. To this end, we choose the coupling matrices in the following form

$$
\begin{aligned}
& F_{a j}^{(1)}=\left(\begin{array}{lll}
0 & -2 \mathbb{N} r & 0
\end{array}\right), F_{i b}^{(2)}=\left(\begin{array}{c}
0 \\
2 \mathbb{N} r \\
0
\end{array}\right), \\
& F_{i j}=\left(\begin{array}{ccc}
\frac{l^{2}}{r^{2}} & 0 & 0 \\
0 & (\beta-1) l^{2} r^{2} & -r\left(l^{2}-2 \mathbb{E}\right) \\
0 & -r\left(l^{2}+2 \mathbb{E}\right) & -l^{2}
\end{array}\right),
\end{aligned}
$$

and $E_{0 a b}=l^{2}$. Then, by utilizing (4.5) one obtains the background of the model in the same forms as (3.12) and (3.22). Analogously, only the $\beta=1$ case of this background with conditions $\mathbb{E}^{2}=l^{4} / 4$ and $\mathbb{N}=0$ can satisfy the vanishing of the one-loop beta-function equations in such a way that the dilaton field is $\Phi=\phi^{(0)}=f z+b$ in which $f^{2}=-1+2 l^{2} \Lambda$.

The corresponding dual background can be cast in the form

$$
\begin{aligned}
d \tilde{s}^{2} & =\frac{l^{2} d r^{2}}{r^{2}}-l^{2} d t^{2}+\frac{1}{l^{2}} d \tilde{x}^{2}+\left((\beta-1) l^{2}+\frac{4 \mathbb{N}^{2}}{l^{2}}\right) r^{2} \\
d \varphi^{2} & -\frac{4 \mathbb{N}}{l^{2}} r d \tilde{x} d \varphi-2 r l^{2} d t d \varphi \\
\tilde{H} & =\mathbb{E} d r \wedge d \varphi \wedge d t,
\end{aligned}
$$

where $\tilde{x}$ is the dualized coordinate of the dual manifold. By taking into account the conditions $\mathbb{E}=l^{2} / 2$ and $\mathbb{N}=0$ one can show that under the transformation $\tilde{x} \rightarrow l^{2} z$, the background (5.17) turns into the same forms as (3.12) and (3.17). In this situation we are faced with self-duality again. Imposing these conditions on (5.17) and putting $\beta=1$ one shows that the dual background is conformally invariant up to the one-loop order in a way that the dual dilaton is found by using Eq. (4.17) to be $\tilde{\Phi}=f z+b-\log l^{2}$.

In addition, we check that the background (5.17) along with a constant dilaton satisfy the vanishing of the two-loop beta-function equations if we have

$\mathbb{E}^{2}=\frac{\beta^{2} l^{4}}{\beta+1}, \quad \mathbb{N}^{2}=\frac{l^{4}}{4}, \quad \alpha^{\prime}=-\frac{(\beta+1) l^{2}}{3 \beta}$,

$$
\tilde{\Lambda}=\frac{5-6 \beta}{6 l^{2}(\beta+1)} .
$$

Also, there is an additional solution with the dilaton field $\tilde{\Phi}=\tilde{f} l^{2} z+b$ provided that

$\mathbb{E}^{2}=\frac{l^{4}}{4}(4 \beta-3), \quad \mathbb{N}=0, \quad \alpha^{\prime}=\frac{2 \beta l^{2}}{5-6 \beta}$,

$\tilde{f}^{2}=-\frac{2}{\beta}+2\left(\tilde{\Lambda} l^{2}+1\right)$.

5.2 Abelian T-duality with two-dimensional Abelian Lie group

\subsubsection{Dualizing with respect to both coordinates $(t, \varphi)$}

In this subsection we shall perform the dualizing on both the coordinates $t$ and $\varphi$. That is, the Lie group $G$ of the target manifold $\mathcal{M} \approx O \times G$ is parameterized by $g=e^{t T_{1}} e^{\varphi T_{2}}$, wherein $\left(T_{1}, T_{2}\right)$ are the basis of the Abelian Lie algebra $\mathcal{G}$ of $G$. So the coordinates of the orbit $O$ are, in this case, represented by $(r, z)$. In this respect, one may choose the background matrices in the following forms

$$
\begin{array}{rlrl}
E_{0 a b} & =\left(\begin{array}{cc}
-l^{2} & -r\left(2 \mathbb{E}+l^{2}\right) \\
r\left(2 \mathbb{E}-l^{2}\right) & (\beta-1) l^{2} r^{2}
\end{array}\right), & F_{a j}^{(1)}=\left(\begin{array}{cc}
0 & 0 \\
0 & 2 \mathbb{N} r
\end{array}\right), \\
F_{i b}^{(2)}=\left(\begin{array}{cc}
0 & 0 \\
0 & -2 \mathbb{N} r
\end{array}\right), & F_{i j}=\left(\begin{array}{cc}
l^{2} & 0 \\
r^{2} & l^{2}
\end{array}\right) .
\end{array}
$$

Hence, the background of the original $\sigma$-model implies the same metric (3.12) and field strength (3.22). From Sect. 3 we know that the $\beta=1$ case of metric (3.12) along with $\mathbb{E}^{2}=l^{4} / 4$ and $\mathbb{N}=0$ can be considered as a solution for the field equations (2.2)-(2.4) up to zeroth order in $\alpha^{\prime}$. In this situation, the matrix $E_{0 a b}$ will no longer be invertible, hence the model can't be conformally invariant at one-loop order.

The corresponding elements to the dual model can be obtained by making use of relations (5.20) and (4.11)-(4.14). They are then read

$$
\begin{aligned}
d \tilde{s}^{2}= & \frac{l^{2} d r^{2}}{r^{2}}+\frac{1}{4 \mathbb{E}^{2}-\beta l^{4}}\left[(\beta-1) l^{2} d \tilde{x}_{1}^{2}-\frac{l^{2}}{r^{2}} d \tilde{x}_{2}^{2}\right. \\
& +\left(4 \mathbb{E}^{2}-4 \mathbb{N}^{2}-\beta l^{4}\right) l^{2} d z^{2}+\frac{2 l^{2}}{r} d \tilde{x}_{1} d \tilde{x}_{2} \\
& \left.+4 \mathbb{N} l^{2}\left(d \tilde{x}_{1} d z-\frac{1}{r} d \tilde{x}_{2} d z\right)\right], \\
\tilde{H}=- & \frac{\mathbb{E}}{r^{2}\left(4 \mathbb{E}^{2}-\beta l^{4}\right)} d \tilde{x}_{1} \wedge d \tilde{x}_{2} \wedge d r,
\end{aligned}
$$

where $\left(\tilde{x}_{1}, \tilde{x}_{2}\right)$ are the dualized coordinates of the dual manifold. We have checked that there is no dilaton field to support the conformality of the dual background (5.21) at one-loop level. But, one can check that the dual background along with 
a constant dilaton field is conformally invariant up to the first order in $\alpha^{\prime}$ provided that

$$
\begin{aligned}
\mathbb{E}^{2} & =\frac{l^{4}}{4}, \quad \mathbb{N}^{2}=\frac{(1-\beta)\left(\beta^{2}+2 \beta-3\right) l^{4}}{3 \beta^{2}+7 \beta-6}, \\
\alpha^{\prime} & =\frac{2 \beta(3 \beta-2) l^{2}}{2 \beta^{2}+\beta-4}, \\
\tilde{\Lambda} & =\frac{2 \beta^{4}-6 \beta^{2}+3 \beta+1}{\beta(3 \beta-2)\left(2 \beta^{2}+\beta-4\right) l^{2}} .
\end{aligned}
$$

Notice that this solution is valid for every $\beta \in(0,2 / 3)$. It should be noted that by taking into consideration $\mathbb{N}=0$ and also condition $\beta \neq 1$ for (5.21) we see that under the transformation

$$
\begin{aligned}
& \tilde{x}_{1}=\sqrt{\frac{\beta l^{4}-4 \mathbb{E}^{2}}{\beta-1} t^{\prime}, \quad \tilde{x}_{2}=\varphi^{\prime},} \\
& r=\frac{1}{r^{\prime} \sqrt{(\beta-1)\left(\beta l^{4}-4 \mathbb{E}^{2}\right)}}, \quad z=z^{\prime},
\end{aligned}
$$

the dual background turns into the same metric (3.12) and field strength (3.17), which this is nothing but the self-duality of the Gödel metrics.

\subsubsection{Dualizing with respect to both coordinates $(t, z)$}

When the dualizing is performed on the coordinates $(t, z)$, it is more appropriate to choose the spectator-dependent matrices as follows:

$$
\begin{aligned}
E_{0 a b} & =\left(\begin{array}{cc}
-l^{2} & 0 \\
0 & l^{2}
\end{array}\right), & F_{a j}^{(1)} & =\left(\begin{array}{cc}
0 & -r\left(2 \mathbb{E}+l^{2}\right) \\
0 & -2 \mathbb{N} r
\end{array}\right), \\
F_{i b}^{(2)} & =\left(\begin{array}{cc}
0 & 0 \\
r\left(2 \mathbb{E}-l^{2}\right) & 2 \mathbb{N} r
\end{array}\right), & F_{i j} & =\left(\begin{array}{cc}
\frac{l^{2}}{r^{2}} & 0 \\
0 & (\beta-1) r^{2} l^{2}
\end{array}\right) .
\end{aligned}
$$

With regard to this choice, we arrive at familiar results, i.e., Eqs. (3.12) and (3.22). According to Sect. 3, the case of $\beta=1$ of this background with conditions $\mathbb{E}^{2}=l^{4} / 4$ and $\mathbb{N}=0$ is a solution for the one-loop beta-function equations in such a way that the dilaton field is obtained to be $\Phi=\phi^{(0)}=f z+b$ in which $f^{2}=-1+2 l^{2} \Lambda$.

Now, one applies formulae (4.11)-(4.14) and (5.24) to obtain the dual background in the following form

$$
\begin{aligned}
d \tilde{s}^{2} & =\frac{l^{2} d r^{2}}{r^{2}}+\frac{1}{l^{2}}\left[-d \tilde{x}_{1}^{2}+d \tilde{x}_{2}^{2}-\left(4 \mathbb{E}^{2}-4 \mathbb{N}^{2}-\beta l^{4}\right)\right. \\
\left.r^{2} d \varphi^{2}+4 r\left(\mathbb{E} d \tilde{x}_{1} d \varphi-\mathbb{N} d \tilde{x}_{2} d \varphi\right)\right] & \\
\tilde{H} & =-\frac{1}{2} d \tilde{x}_{1} \wedge d r \wedge d \varphi
\end{aligned}
$$

Analogously, $\left(\tilde{x}_{1}, \tilde{x}_{2}\right)$ are the dualized coordinates. One can check that only a particular case of the dual background is conformally invariant up to the one-loop order, giving

$$
\begin{aligned}
d \tilde{s}^{2} & =\frac{l^{2} d r^{2}}{r^{2}}-l^{2} d t^{2}+l^{2} d z^{2}-2 r l^{2} d t d \varphi \\
\tilde{H} & =-\frac{l^{2}}{2} d t \wedge d r \wedge d \varphi \\
\tilde{\Phi} & =f z+b-\log l^{4}
\end{aligned}
$$

where $f^{2}=-1+2 l^{2} \Lambda$. We have used the transformation $\tilde{x}_{1} \rightarrow l^{2} t, \tilde{x}_{2} \rightarrow l^{2} z$, and have put $\beta=1, \mathbb{E}=-l^{2} / 2$ and $\mathbb{N}=0$; moreover, the dual dilaton have been obtained by using (4.17).

In addition, a constant dilaton field guaranties the conformal invariance of the dual background (5.25) up to the first order in $\alpha^{\prime}$ if the constants $\mathbb{E}, \mathbb{N}, l, \beta, \tilde{\Lambda}$ and $\alpha^{\prime}$ satisfy the following relations

$$
\begin{aligned}
\mathbb{E}^{2} & =2 l^{4}, \quad \mathbb{N}^{2}=\frac{l^{4}}{24}(\Theta-7 \beta+50), \quad \alpha^{\prime}=\frac{24 \beta l^{2}}{7 \beta-\Theta-20}, \\
\tilde{\Lambda} & =\frac{-59 \beta^{2}+(236+5 \Theta) \beta+20 \Theta-110}{24 \beta l^{2}(\Theta-7 \beta+20)},
\end{aligned}
$$

where $\Theta=\sqrt{\beta^{2}-16 \beta+100}$.

\subsubsection{Dualizing with respect to both coordinates $(\varphi, z)$}

It is also possible to perform the dualizing on both the coordinates $\varphi$ and $z$. So the coordinates of orbit $O$ are chosen to be $(t, r)$. In this way, both the Gödel metrics (3.12) and field strength (3.22) may be yielded from the original $\sigma$-model if one considers

$$
\begin{aligned}
E_{0 a b} & =\left(\begin{array}{cc}
l^{2} & -2 \mathbb{N} r \\
2 \mathbb{N} r & (\beta-1) r^{2} l^{2}
\end{array}\right), & F_{a j}^{(1)} & =\left(\begin{array}{cc}
0 & 0 \\
0 & r\left(2 \mathbb{E}-l^{2}\right)
\end{array}\right), \\
F_{i b}^{(2)} & =\left(\begin{array}{cc}
0 & 0 \\
0 & -r\left(2 \mathbb{E}+l^{2}\right)
\end{array}\right), & F_{i j} & =\left(\begin{array}{cc}
\frac{l^{2}}{r^{2}} & 0 \\
0 & -l^{2}
\end{array}\right) .
\end{aligned}
$$

The one-loop conformal invariance conditions of the Gödel metrics require that we have $\beta=1, \mathbb{E}^{2}=l^{4} / 4$ and $\mathbb{N}=0$. In this situation the $E_{0}$ is singular. Thus, when the dualizing is implemented by the shift of directions $(\varphi, z)$, the pair of the mutually dual models can't be conformally invariant at one-loop level.

Similar to the preceding cases one obtains the corresponding dual $\sigma$-model. If the dualized coordinates are considered to be $\left(\tilde{x}_{1}, \tilde{x}_{2}\right)$, then we have

$$
\begin{aligned}
d \tilde{s}^{2}= & \frac{l^{2} d r^{2}}{r^{2}}+\frac{1}{4 \mathbb{N}^{2}+(\beta-1) l^{4}}\left[(\beta-1) l^{2} d \tilde{x}_{1}^{2}+\frac{l^{2}}{r^{2}} d \tilde{x}_{2}^{2}\right. \\
& \left.+\left(4 \mathbb{E}^{2}-4 \mathbb{N}^{2}-\beta l^{4}\right) l^{2} d t^{2}-4 l^{2}\left(\mathbb{N} d \tilde{x}_{1} d t-\frac{\mathbb{E}}{r} d \tilde{x}_{2} d t\right)\right], \\
\tilde{H}= & \frac{1}{r^{2}\left[4 \mathbb{N}^{2}+(\beta-1) l^{4}\right]}\left(\frac{l^{4}}{2} d r \wedge d \tilde{x}_{2} \wedge d t-\mathbb{N} d r \wedge d \tilde{x}_{1} \wedge d \tilde{x}_{2}\right) .
\end{aligned}
$$

It's worth mentioning that under the transformation $\tilde{x}_{1}=$ $l^{2} z^{\prime}, \tilde{x}_{2}=\varphi^{\prime}, r=1 /\left((\beta-1) l^{2} r^{\prime}\right), t=-t^{\prime}$, the background 
(5.29) can be reduced to the Gödel metrics (3.12) and field strength (3.17); of course when we put $\mathbb{N}=0$ and $\mathbb{E}=l^{2} / 2$.

\subsection{Abelian T-duality with three-dimensional Abelian Lie group}

There is a possibility that we can perform the dualizing on the coordinates $x^{\mu}=(z, t, \varphi)$. In fact, these are the coordinates of the Abelian Lie group of the target space. Choosing the appropriate spectator-dependent matrices in the forms

$$
\begin{aligned}
& E_{0 a b}=\left(\begin{array}{ccc}
l^{2} & 0 & -2 \mathbb{N} r \\
0 & -l^{2} & -\left(l^{2}+2 \mathbb{E}\right) r \\
2 \mathbb{N} r-\left(l^{2}-2 \mathbb{E}\right) r & (\beta-1) l^{2} r^{2}
\end{array}\right), \\
& F_{a j}^{(1)}=0, \quad F_{i b}^{(2)}=0, \quad F_{i j}=\frac{l^{2}}{r^{2}},
\end{aligned}
$$

one gets the coupling matrices of the original $\sigma$-model. Then, by making use of (4.5) the metric and field strength of the model are obtained to be of the same forms as (3.12) and (3.22), respectively. Because of the invertibility of the matrix $E_{0}$, we can't accept the values $\beta=1, \mathbb{E}^{2}=l^{4} / 4$ and $\mathbb{N}=0$ for $E_{0}$. Accordingly, the original $\sigma$-model doesn't satisfy the one-loop beta-function equations.

The dual background can be cast in the form

$$
\begin{aligned}
d \tilde{s}^{2}= & \frac{l^{2}}{r^{2}} d r^{2}-\frac{1}{4\left(\mathbb{E}^{2}-\mathbb{N}^{2}\right)-\beta l^{4}}\left[\left(\beta l^{2}-\frac{4 \mathbb{E}^{2}}{l^{2}}\right) d \tilde{x}_{1}^{2}\right. \\
& -\left((\beta-1) l^{2}+\frac{4 \mathbb{N}^{2}}{l^{2}}\right) d \tilde{x}_{2}^{2}+\frac{l^{2}}{r^{2}} d \tilde{x}_{3}^{2}+\frac{8 \mathbb{E} \mathbb{N}}{l^{2}} d \tilde{x}_{1} d \tilde{x}_{2} \\
& \left.-\frac{2 l^{2}}{r} d \tilde{x}_{2} d \tilde{x}_{3}\right], \\
\tilde{H}= & -\frac{1}{r^{2}\left(4\left(\mathbb{E}^{2}-\mathbb{N}^{2}\right)-\beta l^{4}\right)}\left[\mathbb{E} d r \wedge d \tilde{x}_{2} \wedge d \tilde{x}_{3}\right. \\
& \left.+\mathbb{N} d r \wedge d \tilde{x}_{3} \wedge d \tilde{x}_{1}\right],
\end{aligned}
$$

where $\left(\tilde{x}_{1}, \tilde{x}_{2}, \tilde{x}_{3}\right)$ are the dualized coordinates. One can easily check that the dual background is not also conformally invariant up to the one-loop order. It is quite interesting to comment on the $\beta=1 / 2$ case of the background (5.31). For this case, one can easily show that the field equations (2.2)-(2.4) are satisfied with a constant dilaton provided that

$$
\mathbb{E}=0, \quad \mathbb{N}^{2}=\frac{l^{4}}{8}, \quad \alpha^{\prime}=4 l^{2}, \quad \tilde{\Lambda}=\frac{3}{8 l^{2}} .
$$

Putting $\mathbb{N}=0$ in both the metric and corresponding $B$-field of the background (5.31) and then utilizing the transformation

$$
\tilde{x}_{1}=l^{2} z^{\prime}, \quad \tilde{x}_{2}=\sqrt{\frac{\beta l^{4}-4 \mathbb{E}^{2}}{\beta-1}} t^{\prime},
$$

$$
\tilde{x}_{3}=\sqrt{(\beta-1)\left(\beta l^{4}-4 \mathbb{E}^{2}\right)} \varphi^{\prime}, \quad r=\frac{1}{r^{\prime}},
$$

the dual background turns into the same metric (3.12) and field strength (3.17).

In summary, we obtained seven Abelian duals for the Gödel spacetimes and showed that they were self-dual when $\mathbb{N}$ went to zero, and in some instances, one must consider $\mathbb{E}=l^{2} / 2$. The results of this section are clearly summarized in Table 1. There, we have shown the backgrounds solving the one-loop equations, and solve also the two-loop equations without $\alpha^{\prime}$-corrections.

Before closing this section, we note the fact that our Abelian dual models were obtained by the PL T-duality transformations at the classical level. The PL T-duality transformations are valid only at the one-loop level. Our results showed that by applying the rules of Abelian T-duality without further corrections, we were still able to obtain two-loop solutions.

\section{Abelian T-duality of Gödel string cosmologies up to $\alpha^{\prime}$-corrections}

In this section, we study the Abelian T-duality of Gödel string cosmologies up to $\alpha^{\prime}$-corrections when the dualizing is implemented by the shift of directions $z$ and $t$. To this end, we use the T-duality rules at two-loop order derived by KM [43]. Before proceeding further, let us review the $\alpha^{\prime}$-corrected T-duality rules of KM.

\subsection{The $\alpha^{\prime}$-corrections from T-duality rules at two-loop}

As mentioned in the introduction section, the authors of Ref. [43] had obtained the two-loop $\sigma$-model corrections to the T-duality map in string theory by using the effective action approach. They had found the explicit form for the $\mathcal{O}\left(\alpha^{\prime}\right)$ modifications of the lowest order duality transformations by focusing on backgrounds that have a single Abelian isometry. Recently, it has been shown that [57] using the $\alpha^{\prime}$-corrected T-duality rules of [43] one can obtain explicit $\alpha^{\prime}$-corrections for Yang-Baxter deformed models. In fact, it has been argued that (homogeneous) Yang-Baxter deformed string $\sigma$-models that are conformal at one loop remain conformal at two loops.

Similar to Ref. [57] we follow [43] and choose the reduced metric $g_{\mu \nu}$, antisymmetric field $b_{\mu \nu}$ and dilaton $\Phi$ of the $d$ dimensional spacetime, respectively, according to

$$
\begin{aligned}
d s^{2}= & G_{M N} d X^{M} d X^{N}=g_{\mu \nu} d x^{\mu} d x^{\nu} \\
& +e^{2 \sigma}\left(d \underline{x}+V_{\mu} d x^{\mu}\right)^{2} \\
B= & \frac{1}{2} B_{M N} d X^{M} \wedge d X^{N}=\frac{1}{2} b_{\mu \nu} d x^{\mu} \wedge d x^{\nu} \\
& +\frac{1}{2} W_{\mu} V_{\nu} d x^{\mu} \wedge d x^{\nu}+W_{\mu} d x^{\mu} \wedge d \underline{x},
\end{aligned}
$$




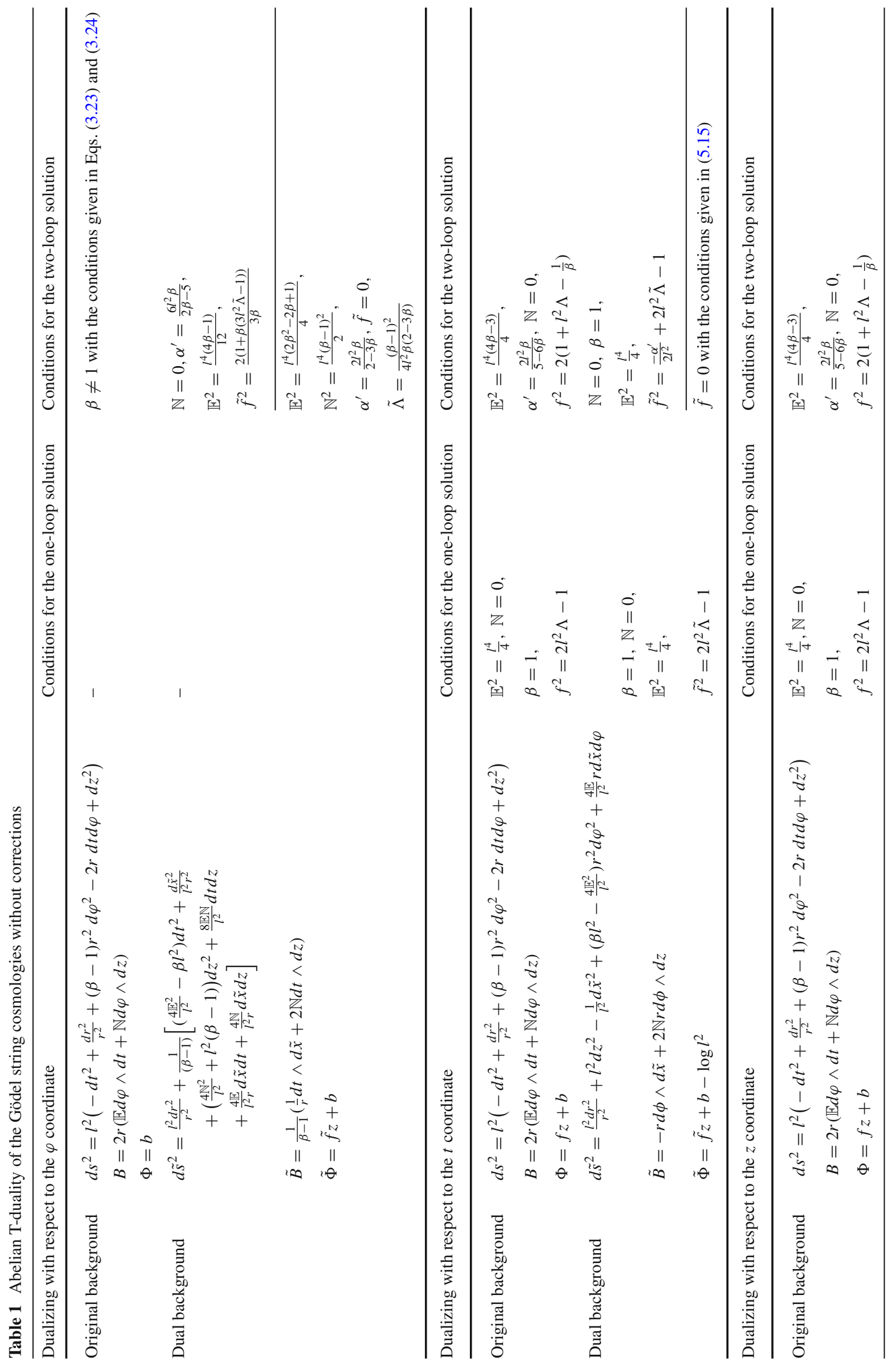




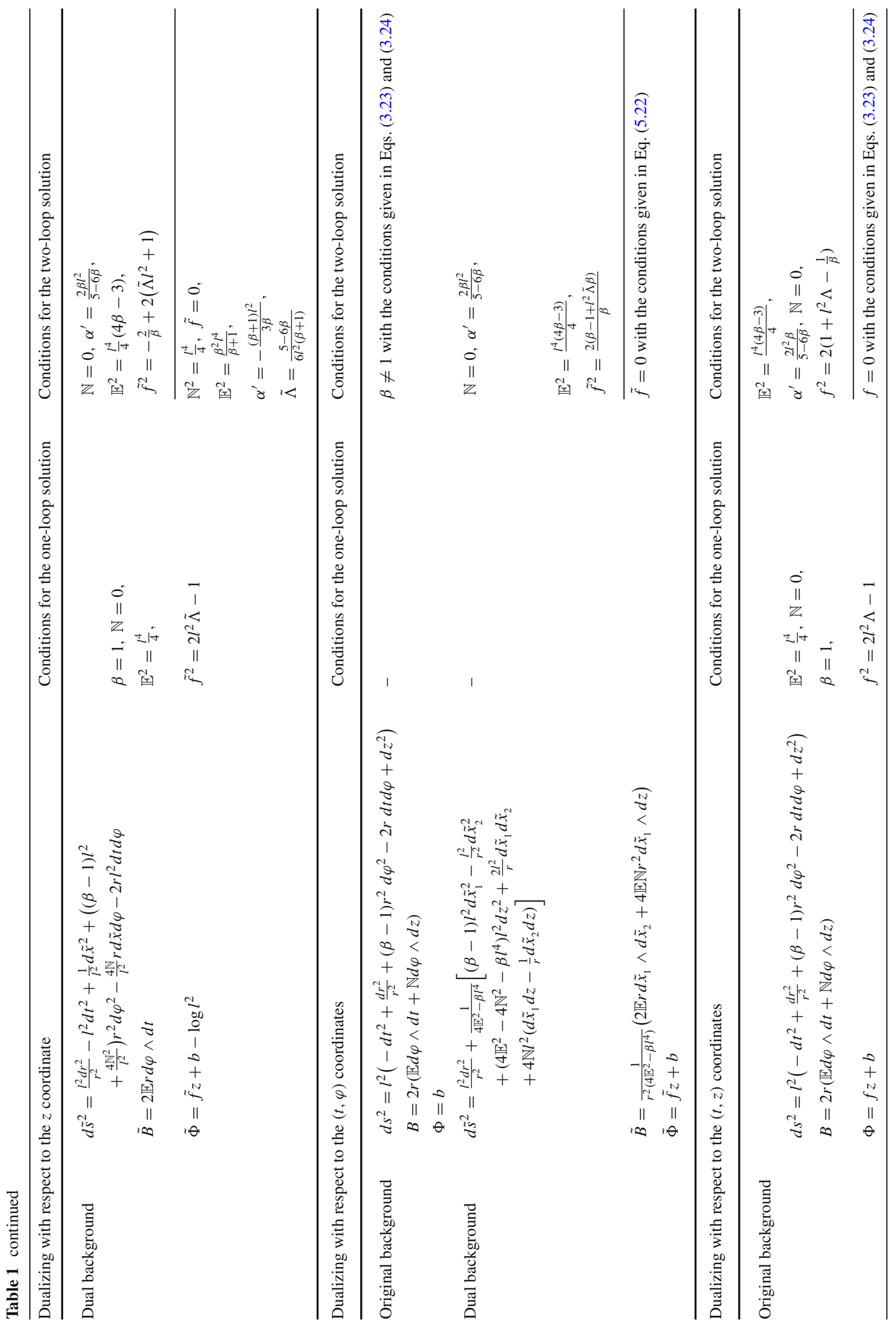




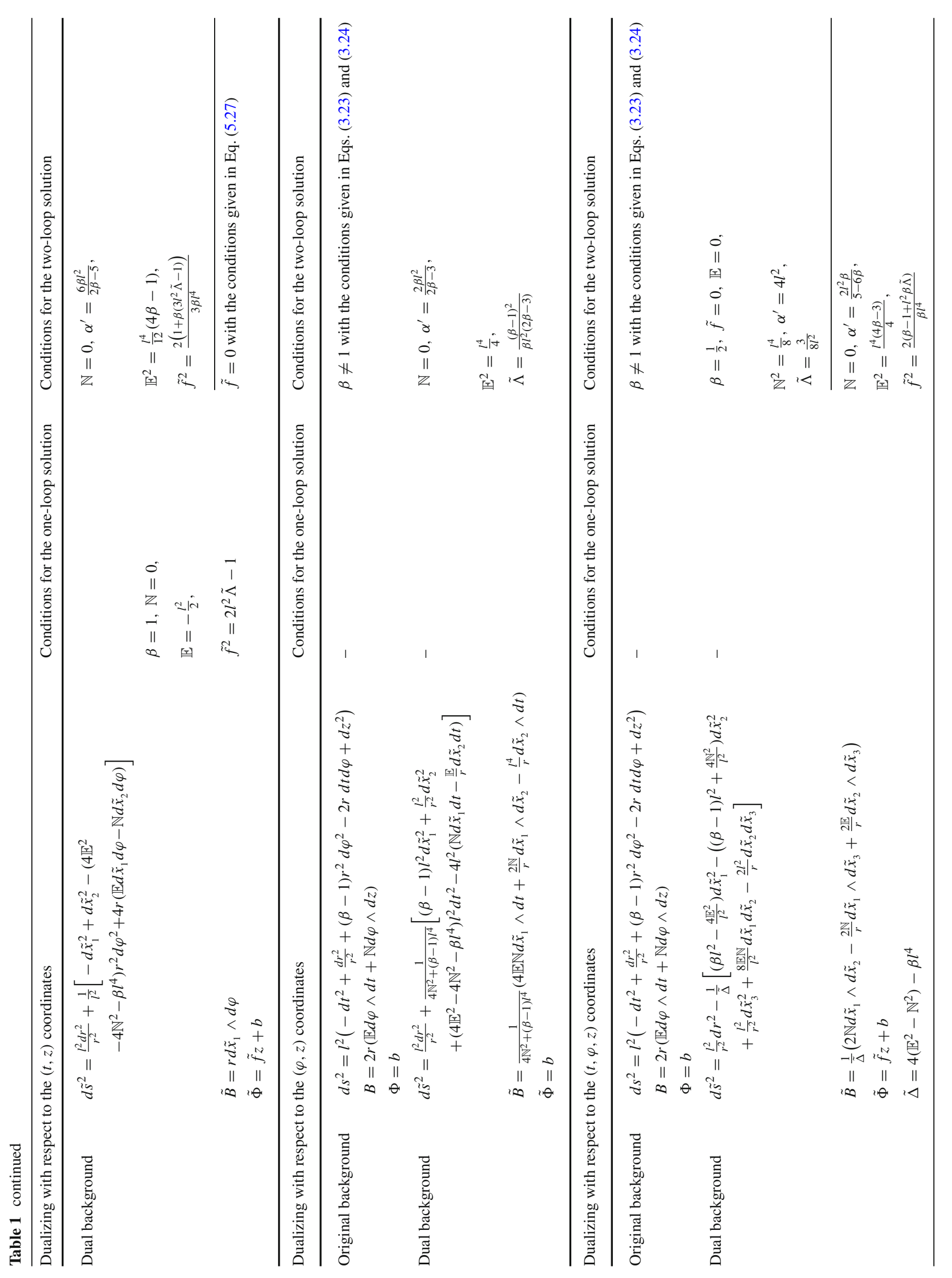




$$
\Phi=\hat{\phi}+\frac{1}{2} \sigma
$$

where the field $V_{\mu}$ is the standard Kaluza-Klein gauge field coupling to the momentum modes of the theory, and $W_{\mu}$ is the other gauge field, which couples to the winding modes. Here we assume that the isometry we want to dualize is simply implemented by a shift of a coordinate, that we denote by $\underline{x}$. We have also introduced the scalars $\sigma$ and $\hat{\phi}$. In components, the relations to identify the fields of the dimensional reduction are

$$
\begin{aligned}
g_{\mu \nu} & =G_{\mu \nu}-\frac{G_{\mu \underline{x}} G_{\nu \underline{x}}}{G_{\underline{x x}}}, \quad b_{\mu \nu}=B_{\mu \nu}+\frac{G_{\underline{x}[\mu} B_{\nu] \underline{x}}}{G_{\underline{x x}}}, \\
V_{\mu} & =\frac{G_{\mu \underline{x}}}{G_{\underline{x x}}}, \quad W_{\mu}=B_{\mu \underline{x}}, \\
\sigma & =\frac{1}{2} \log G_{\underline{x \underline{x}}} .
\end{aligned}
$$

In order to apply the T-duality rules of KM to our purpose, we will therefore first need to implement the field redefinitions to go from Hull and Townsend (HT) scheme ${ }^{3}$ [48] to that of KM. This work has been done in [57]. The field redefinitions that we will use are

$$
\begin{aligned}
& G_{M N}^{(H T)}=G_{M N}^{(K M)}+\alpha^{\prime}\left(\mathcal{R}_{M N}-\frac{1}{2} H_{M N}^{2}\right), \\
& B_{M N}^{(H T)}=B_{M N}^{(K M)}+\alpha^{\prime}\left(-H_{M N P} \nabla^{P} \Phi\right), \\
& \Phi^{(H T)}=\Phi^{(K M)}+\alpha^{\prime}\left(-\frac{3}{32} H^{2}+\frac{1}{8} \mathcal{R}-\frac{1}{2}(\nabla \Phi)^{2}\right) .
\end{aligned}
$$

Thus, the two-loop T-duality transformation equations in the KM scheme are given by [43]

$$
\begin{aligned}
\tilde{\sigma}= & -\sigma+\alpha^{\prime}\left[(\nabla \sigma)^{2}+\frac{1}{8}\left(e^{2 \sigma} Z+e^{-2 \sigma} T\right)\right], \\
\tilde{V}_{\mu}= & W_{\mu}+\alpha^{\prime}\left[W_{\mu \nu} \nabla^{\nu} \sigma+\frac{1}{4} h_{\mu \nu \rho} V^{\nu \rho} e^{2 \sigma}\right], \\
\tilde{W}_{\mu}= & V_{\mu}+\alpha^{\prime}\left[V_{\mu \nu} \nabla^{\nu} \sigma-\frac{1}{4} h_{\mu \nu \rho} W^{\nu \rho} e^{-2 \sigma}\right], \\
\tilde{b}_{\mu \nu}= & b_{\mu \nu}+\alpha^{\prime}\left[V_{\rho[\mu} W_{\nu]}^{\rho}+\left(W_{[\mu \rho} \nabla^{\rho} \sigma\right.\right. \\
& \left.+\frac{1}{4} e^{2 \sigma} h_{[\mu \rho \lambda} V^{\rho \lambda}\right) V_{\nu]} \\
& \left.+\left(V_{[\mu \rho} \nabla^{\rho} \sigma-\frac{1}{4} e^{-2 \sigma} h_{[\mu \rho \lambda} W^{\rho \lambda}\right) W_{\nu]}\right] .
\end{aligned}
$$

These transformations are written using the following definitions

$$
\begin{aligned}
W_{\mu \nu} & =\partial_{\mu} W_{\nu}-\partial_{\nu} W_{\mu}, \\
V_{\mu \nu} & =\partial_{\mu} V_{\nu}-\partial_{\nu} V_{\mu}, \\
h_{\mu \nu \rho} & =H_{\mu \nu \rho}-3 W_{[\mu \nu} V_{\rho]} .
\end{aligned}
$$

\footnotetext{
${ }^{3}$ Notice that here we use the two-loop beta-function equations of the HT scheme which were used in [57].
}

In addition,

$$
\begin{aligned}
Z_{\mu \nu} & =V_{\mu \rho} V_{\nu}{ }^{\rho}, & Z & =Z_{\mu}^{\mu}, \\
T_{\mu \nu} & =W_{\mu \rho} W_{\nu}{ }^{\rho}, & & T=T_{\mu}^{\mu} .
\end{aligned}
$$

All the lowering and raising of the indices will be done with respect to the reduced metric $g_{\mu \nu}$.

\subsubsection{Abelian T-duality with respect to the $z$ coordinate up to $\alpha^{\prime}$-corrections}

In Sect. 3 we showed that the $\beta=1$ case of the Gödel spacetimes with field strength $H_{t r \varphi}=l^{2} / 2$ and a constant dilaton field satisfy the beta-function equations up to the first order in $\alpha^{\prime}$ so that the relation between constants $\Lambda, l$ and $\alpha^{\prime}$ may be expressed as $\Lambda=1 / 2 l^{2}+\alpha^{\prime} /\left(4 l^{4}\right)$. Here we use the conditions for two-loop conformal invariance of the bosonic string $\sigma$-model in the HT scheme which were used in [57]. In the HT scheme, the aforementioned solution can be written as $^{4}$

$$
\begin{aligned}
d s^{2^{(H T)}} & =-l^{2} d t^{2}+\frac{l^{2} d r^{2}}{r^{2}}-2 r l^{2} d t d \varphi+l^{2} d z^{2}, \\
H^{(H T)} & =l^{2} d t \wedge d r \wedge d \varphi, \\
\Phi^{(H T)} & =\frac{b}{2} .
\end{aligned}
$$

One can use the field redefinitions in Eqs. (6.7)-(6.9) to write the above solution in the KM scheme, expressing

$$
\begin{aligned}
d s^{2^{(K M)}=} & -l^{2} d t^{2}+\frac{l^{2} d r^{2}}{r^{2}}-2 r l^{2} d t d \varphi+l^{2} d z^{2} \\
& +\alpha^{\prime}\left(\frac{1}{2} d t^{2}-\frac{d r^{2}}{2 r^{2}}+r d t d \varphi\right), \\
H^{(K M)}= & l^{2} d t \wedge d r \wedge d \varphi, \\
\Phi^{(K M)}= & \frac{b}{2}-\frac{3}{8 l^{2}} \alpha^{\prime} .
\end{aligned}
$$

Notice that the isometry we want to dualize is that the shift of the $z$ coordinate, i.e., $\underline{x}=z$. Comparing (6.20) with Eqs. (6.1)-(6.3) or using (6.4)-(6.6) one concludes that all components of $V_{\mu}$ and $W_{\mu}$ are zero; consequently, $V_{\mu \nu}=0, W_{\mu \nu}=0, Z=0$ and $T=0$. Furthermore,

$\sigma^{(K M)}=\frac{1}{2} \ln l^{2}, \quad \hat{\phi}=\Phi^{(K M)}-\frac{1}{4} \ln l^{2}$.

Applying these results to the two-loop T-duality transformation Eqs. (6.10)-(6.13) one can get the dual solution in the KM scheme. Finally, by employing field redefinitions in Eqs. (6.7)-(6.9) we can write the dual solution in the HT scheme, giving

$\tilde{d} s^{2^{(H T)}}=-l^{2} d t^{2}+\frac{l^{2} d r^{2}}{r^{2}}-2 r l^{2} d t d \varphi+\frac{1}{l^{2}} d \underline{\tilde{x}}^{2}$,

\footnotetext{
4 To go from our conventions to those of the HT [57] one can send $\Phi / 2 \rightarrow \Phi^{(H T)}$ and $2 H \rightarrow H^{(H T)}$.
} 


$$
\begin{aligned}
& \tilde{H}^{(H T)}=l^{2} d t \wedge d r \wedge d \varphi, \\
& \tilde{\Phi}^{(H T)}=\frac{b}{2}-\frac{1}{2} \log l^{2} .
\end{aligned}
$$

It should be noted that utilizing the transformation $\underline{\tilde{x}} \rightarrow l^{2} z$ and shifting $\frac{b}{2} \rightarrow \frac{b}{2}+\frac{1}{2} \log l^{2}$, the dual solution (6.22) turns into the original one. Hence, we have shown that the Abelian T-duals generated by Gödel spacetimes at two-loop order are self-dual.

\subsubsection{Abelian T-duality with respect to the t coordinate up to $\alpha^{\prime}$-corrections}

Another solution for the two-loop beta-function equations in the HT scheme including the $\beta=1$ case of the Gödel spacetimes with a non-constant dilaton field is given by

$$
\begin{aligned}
d s^{2^{(H T)}} & =-l^{2} d t^{2}+\frac{l^{2} d r^{2}}{r^{2}}-2 r l^{2} d t d \varphi+l^{2} d z^{2}, \\
H^{(H T)} & =-l^{2} d t \wedge d r \wedge d \varphi \\
\Phi^{(H T)} & =a z-\alpha^{\prime} \frac{z}{16 a l^{2}}
\end{aligned}
$$

where $4 a^{2}=-1+2 l^{2} \Lambda$. In what follows we want to perform the dualizing on the time coordinate. Since $G_{\underline{x x}}=G_{t t}$ is negative, we are faced with a timelike case. In principle, the rules of T-duality are derived assuming that the coordinate to be dualized is spacelike. Formally, we can achieve that in the timelike case if we first do the analytic continuation $t \rightarrow i t^{\prime}$, apply T-duality as usual and then go back doing $t^{\prime} \rightarrow-i t$. If one just looks at the NS-NS sector (metric, Bfield, dilaton), this is equivalent to applying the rules without the analytic continuation, i.e. with $G_{\underline{x x}}$ negative. The result that he obtains is the same. In the type $I I$ string theory case, the story is more subtle. As argued in [58], the timelike Tduals of the $I I A$ and $I I B$ string theories are new theories that referred to as the $I I B^{*}$ and $I I A^{*}$ string theories, respectively.

However, by using the Wick rotations $t \rightarrow i t^{\prime}$ and $\varphi \rightarrow$ $i \varphi^{\prime}$ the solution (6.23) is written as

$$
\begin{aligned}
d s^{2^{(H T)}} & =l^{2} d t^{\prime 2}+\frac{l^{2} d r^{2}}{r^{2}}+2 r l^{2} d t^{\prime} d \varphi^{\prime}+l^{2} d z^{2}, \\
H^{(H T)} & =l^{2} d t^{\prime} \wedge d r \wedge d \varphi^{\prime} \\
\Phi^{(H T)} & =a z-\alpha^{\prime} \frac{z}{16 a l^{2}} .
\end{aligned}
$$

Accordingly, the isometry we want to dualize is spacelike. That is the shift of the $t^{\prime}$ coordinate, i.e., $x=t^{\prime}$. By using the field redefinitions in Eqs. (6.7)-(6.9) one can write the solution (6.24) in the KM scheme, giving

$$
\begin{aligned}
d s^{2^{(K M)}}= & l^{2} d t^{\prime 2}+\frac{l^{2} d r^{2}}{r^{2}}+2 r l^{2} d t^{\prime} d \varphi^{\prime}+l^{2} d z^{2} \\
& -\alpha^{\prime}\left(\frac{1}{2} d t^{\prime 2}+\frac{d r^{2}}{2 r^{2}}+r d t^{\prime} d \varphi^{\prime}\right),
\end{aligned}
$$

$$
\begin{aligned}
& H^{(K M)}=l^{2} d t^{\prime} \wedge d r \wedge d \varphi^{\prime}, \\
& \Phi^{(K M)}=a z+\left(\frac{8 a^{3}-6 a-z}{16 a l^{2}}\right) \alpha^{\prime} .
\end{aligned}
$$

Comparing (6.25) with Eqs (6.1)-(6.3) or using (6.4)-(6.6) one obtains that only the nonzero components of $V_{\mu}$ and $W_{\mu}$ are

$V_{\varphi^{\prime}}=r, \quad W_{\varphi^{\prime}}=r l^{2}$.

In addition,

$$
\begin{aligned}
\sigma^{(K M)} & =\frac{1}{2} \log \left(l^{2}-\frac{\alpha^{\prime}}{2}\right)=\frac{1}{2} \log l^{2}-\frac{1}{4 l^{2}} \alpha^{\prime}+\mathcal{O}\left(\alpha^{\prime 2}\right), \\
\hat{\phi} & =\Phi^{(K M)}-\frac{1}{4} \log l^{2}+\frac{1}{8 l^{2}} \alpha^{\prime}+\mathcal{O}\left(\alpha^{\prime 2}\right) .
\end{aligned}
$$

Using the above results, $Z, T, h_{\mu \nu \rho}$, and only the nonzero components of $V_{\mu \nu}$ and $W_{\mu \nu}$ are derived to be

$$
\begin{aligned}
Z & =-\frac{2}{l^{4}}\left(1-\frac{\alpha^{\prime}}{2 l^{2}}\right)^{-2}, \quad T=-2\left(1-\frac{\alpha^{\prime}}{2 l^{2}}\right)^{-2}, \\
h_{\mu \nu \rho} & =0, \quad V_{r \varphi^{\prime}}=1, \quad W_{r \varphi^{\prime}}=l^{2} .
\end{aligned}
$$

In order to calculate the dual background up to $\alpha^{\prime}$-corrections one can substitute Eqs. (6.26)-(6.28) into the two-loop Tduality transformation Eqs. (6.10)-(6.13). Thus, using Eqs. (6.1)-(6.3), the dual solution is obtained in the KM scheme to be of the form

$$
\begin{aligned}
\tilde{d} s^{2^{(K M)}=} & \frac{1}{l^{2}} d \underline{\tilde{x}}^{2}+\frac{l^{2} d r^{2}}{r^{2}}+2 r d \underline{\tilde{x}} d \varphi^{\prime}+l^{2} d z^{2} \\
& -\alpha^{\prime}\left(\frac{1}{2 l^{4}} d \underline{\tilde{x}^{2}}+\frac{d r^{2}}{2 r^{2}}+\frac{r}{l^{2}} d \underline{\tilde{x}} d \varphi^{\prime}\right), \\
\tilde{H}^{(K M)}= & d \underline{\tilde{x}} \wedge d r \wedge d \varphi^{\prime}, \\
\tilde{\Phi}^{(K M)}= & -\frac{1}{2} \log l^{2}+a z+\left(\frac{8 a^{3}-6 a-z}{16 a l^{2}}\right) \alpha^{\prime} .
\end{aligned}
$$

Finally, one can apply Eqs. (6.7)-(6.9) to write the dual solution (6.29) in the HT scheme. It is then read

$$
\begin{aligned}
\tilde{d} s^{2^{(H T)}} & =\frac{1}{l^{2}} d \underline{\tilde{x}}^{2}+\frac{l^{2} d r^{2}}{r^{2}}+2 r d \underline{\tilde{x}} d \varphi^{\prime}+l^{2} d z^{2}, \\
\tilde{H}^{(H T)} & =d \underline{\tilde{x}} \wedge d r \wedge d \varphi^{\prime}, \\
\tilde{\Phi}^{(H T)} & =-\frac{1}{2} \log l^{2}+a z-\frac{z}{16 a l^{2}} \alpha^{\prime} .
\end{aligned}
$$

As shown we used the Wick rotations $t \rightarrow i t^{\prime}$ and $\varphi \rightarrow i \varphi^{\prime}$, then, applied the rules of T-duality as usual in order to obtain the dual solution at two-loop order. Now, one can go back doing the analytic continuation $\underline{\tilde{x}} \rightarrow-i t$ and $\varphi^{\prime} \rightarrow-i \varphi$. We represent the results of Abelian T-duality of the Gödel string cosmologies up to $\alpha^{\prime}$-corrections in Table 2 . 


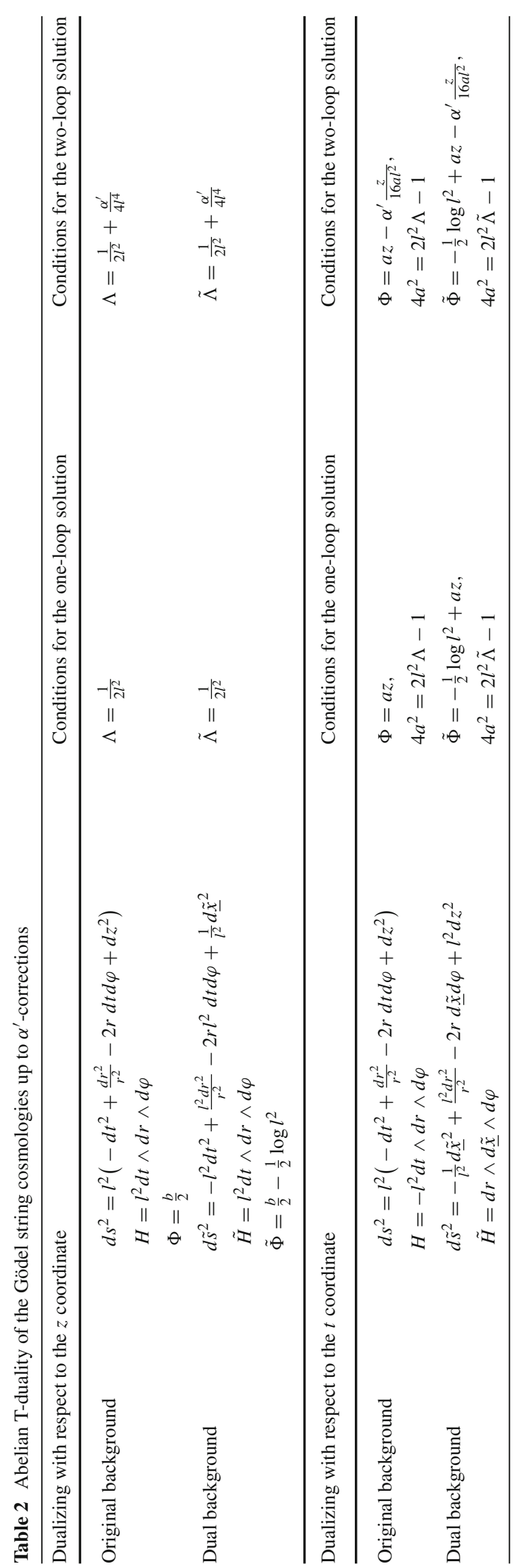




\section{Non-Abelian T-dualization of Gödel string cosmologies}

We now wish to apply the formulation of Sect. 4 in order to study the non-Abelian T-dualization of the Gödel spacetimes. In this section, we explicitly construct some of the PL T-dual $\sigma$-models on $2+2$ - and $3+1$-dimensional target manifolds. In any case, the metrics of the original $\sigma$-models represent the Gödel spacetimes. From the analysis of Sect. 3, it is followed that the backgrounds of the models are conformally invariant up to two-loop order such that they satisfy Eqs. (2.2)-(2.4). We clarify the corresponding dual spacetimes structure, as well as the conformal invariance conditions of them. In a particular case of the models, we study the PL self-duality of $A d S_{3} \times \mathbb{R}$ space.

\subsection{Non-Abelian T-duality from a $2+2$-dimensional manifold with the $A_{2}$ Lie group}

As discussed in Ref. [39], the 2+2-dimensional manifold $\mathcal{M} \approx O \times G$ with two-dimensional real non-Abelian Lie group $G=A_{2}$ is wealthy. We shall obtain the Gödel spacetimes from a T-dualizable $\sigma$-model constructed out of the $2+2$-dimensional manifold $\mathcal{M} \approx O \times G$ where $G$ acts freely on $\mathcal{M}$, while $O$ is the orbit of $G$ in $\mathcal{M}$. In this way, the non-Abelian T-duality of the Gödel spacetimes is studied here. The dual Lie group $\tilde{G}$ acting freely on the dual manifold $\tilde{\mathcal{M}} \approx O \times \tilde{G}$ is considered to be two-dimensional Abelian Lie group $2 A_{1}$. The Lie algebras of the Lie groups $G$ and $\tilde{G}$ are denoted by $\mathcal{A}_{2}$ and $2 \mathcal{A}_{1}$, respectively. As mentioned in Sect. 4, having Drinfeld doubles one can construct PL Tdual $\sigma$-models on them. The four-dimensional Lie algebra of the Drinfeld double $\left(\mathcal{A}_{2}, 2 \mathcal{A}_{1}\right)$ is defined by the following nonzero commutation relations:

$\left[T_{1}, T_{2}\right]=T_{2},\left[T_{1}, \tilde{T}^{2}\right]=-\tilde{T}^{2},\left[T_{2}, \tilde{T}^{2}\right]=\tilde{T}^{1}$,

where $\left(T_{1}, T_{2}\right)$ and $\left(\tilde{T}^{1}, \tilde{T}^{2}\right)$ are the basis of $\mathcal{A}_{2}$ and $2 \mathcal{A}_{1}$, respectively.

In order to write the action of the $\sigma$-models (4.5) and (4.10) on the $2+2$-dimensional manifolds $\mathcal{M}$ and $\tilde{\mathcal{M}}$ we need to calculate the components of the right-invariant MaurerCartan forms $R_{ \pm}^{a}$ and $\tilde{R}_{ \pm_{a}}$ on the Lie groups $A_{2}$ and $2 A_{1}$, respectively. To this end, we parametrize elements of the $A_{2}$ and $2 A_{1}$ as

$g=e^{x_{1} T_{1}} e^{x_{2} T_{2}}, \quad \tilde{g}=e^{\tilde{x}_{1} \tilde{T}^{1}} e^{\tilde{x}_{2} \tilde{T}^{2}}$,

where $\left(x_{1}, x_{2}\right)$ and $\left(\tilde{x}_{1}, \tilde{x}_{2}\right)$ stand for the coordinates of the Lie groups $A_{2}$ and $2 A_{1}$, respectively. $R_{ \pm}^{a}$ 's and $\tilde{R}_{ \pm_{a}}$ 's are then obtained to be of the forms

$$
R_{ \pm}^{1}=\partial_{ \pm} x_{1}, \quad R_{ \pm}^{2}=e^{x_{1}} \partial_{ \pm} x_{2},
$$

$\tilde{R}_{ \pm_{1}}=\partial_{ \pm} \tilde{x}_{1}, \quad \tilde{R}_{ \pm_{2}}=\partial_{ \pm} \tilde{x}_{2}$

For our purpose it is also necessary to compute the matrices $\Pi^{a b}(g)$ and $\tilde{\Pi}_{a b}(\tilde{g})$. Using Eqs. (7.1) and (7.2) and also applying Eq. (4.4) for both the Lie groups $A_{2}$ and $2 A_{1}$, we get

$\Pi^{a b}(g)=0, \quad \tilde{\Pi}_{a b}(\tilde{g})=\left(\begin{array}{cc}0 & -\tilde{x}_{2} \\ \tilde{x}_{2} & 0\end{array}\right)$.

Let us now choose the spectator-dependent background matrices as

$$
\begin{aligned}
E_{0 a b} & =\left(\begin{array}{cc}
l^{2} & 0 \\
0 & l^{2}(\beta-1)
\end{array}\right), & F_{a j}^{(1)} & =\left(\begin{array}{cc}
0 & 0 \\
2 \mathbb{E}-l^{2} & 2 \mathbb{N}
\end{array}\right), \\
F_{i b}^{(2)} & =\left(\begin{array}{cc}
0 & -\left(2 \mathbb{E}+l^{2}\right) \\
0 & -2 \mathbb{N}
\end{array}\right), & F_{i j} & =\left(\begin{array}{cc}
-l^{2} & 0 \\
0 & l^{2}
\end{array}\right) .
\end{aligned}
$$

Inserting (7.6) into Eqs. (4.6)-(4.9) and then utilizing formulas (7.3) and (7.5) together with (4.5), the original $\sigma$-model is worked out to be

$$
\begin{aligned}
S= & \frac{1}{2} \int d \sigma^{+} d \sigma^{-}\left[l^{2} \partial_{+} x_{1} \partial_{-} x_{1}+l^{2}(\beta-1) e^{2 x_{1}} \partial_{+} x_{2} \partial_{-} x_{2}\right. \\
& +\left(2 \mathbb{E}-l^{2}\right) e^{x_{1}} \partial_{+} x_{2} \partial_{-} y_{1} \\
& -\left(2 \mathbb{E}+l^{2}\right) e^{x_{1}} \partial_{+} y_{1} \partial_{-} x_{2}+2 \mathbb{N} e^{x_{1}}\left(\partial_{+} x_{2} \partial_{-} y_{2}-\partial_{+} y_{2} \partial_{-} x_{2}\right) \\
& \left.-l^{2} \partial_{+} y_{1} \partial_{-} y_{1}+l^{2} \partial_{+} y_{2} \partial_{-} y_{2}\right]-\frac{1}{4 \pi} \int d \sigma^{+} d \sigma^{-} R^{(2)} \Phi(X),
\end{aligned}
$$

where $y^{i}=\left(y_{1}, y_{2}\right)$ are the coordinates of the orbit $O$ of $G$ in manifold $\mathcal{M}$. To be more specific, we use the coordinate transformation

$e^{x_{1}}=r, \quad x_{2}=\varphi, \quad y_{1}=t, \quad y_{2}=z$.

Then, by identifying the action (7.7) with the $\sigma$-model of the form (2.1), the corresponding line element and antisymmetric tensor can be, respectively, cast in the forms

$$
\begin{aligned}
d s^{2} & =l^{2}\left[-d t^{2}+\frac{d r^{2}}{r^{2}}+(\beta-1) r^{2} d \varphi^{2}-2 r d t d \varphi+d z^{2}\right] \\
B & =2 \mathbb{E} r d \varphi \wedge d t+2 \mathbb{N} r d \varphi \wedge d z .
\end{aligned}
$$

The line element (7.9) is nothing but the Gödel metric (3.12), and the field strength corresponding to the $B$-field (7.10) is easily obtained to be the same form as (3.22). Because of the invertibility of the matrix $E_{0}$, the parameter $\beta$ must be here considered to be different from one. Accordingly, the model (7.7) will not be conformally invariant at one-loop level. As discussed in Sect. 3 (class B of solutions), the metric (7.9) and field strength (3.22) together with a constant dilaton field satisfy the two-loop beta-function Eqs. (2.2)-(2.4).

In order to construct the dual $\sigma$-model corresponding to the model (7.7) we use the action (4.10). The dual coupling 
matrices can be obtained by inserting (7.5) and (7.6) into (4.11)-(4.14). They are then read

$$
\begin{aligned}
& \tilde{E}^{a b}=\frac{1}{\Delta}\left(\begin{array}{cc}
l^{2}(\beta-1) & \tilde{x}_{2} \\
-\tilde{x}_{2} & l^{2}
\end{array}\right), \\
& \tilde{\phi}_{i j}=\left(\begin{array}{cc}
-l^{2}\left[1-\frac{1}{\Delta}\left(4 \mathbb{E}^{2}-l^{4}\right)\right] & \frac{2 \mathbb{N} l^{2}}{\Delta}\left(2 \mathbb{E}+l^{2}\right) \\
\frac{2 \mathbb{N} l^{2}}{\Delta}\left(2 \mathbb{E}-l^{2}\right) & l^{2}\left(1+\frac{4 \mathbb{N}^{2}}{\Delta}\right)
\end{array}\right), \\
& \tilde{\phi}^{(1)^{a}}{ }_{j}=\frac{1}{\Delta}\left(\begin{array}{cc}
\left(2 \mathbb{E}-l^{2}\right) \tilde{x}_{2} & 2 \mathbb{N} \tilde{x}_{2} \\
\left(2 \mathbb{E}-l^{2}\right) l^{2} & 2 \mathbb{N} l^{2}
\end{array}\right), \\
& \tilde{\phi}_{i}^{(2)^{b}}=\frac{1}{\Delta}\left(\begin{array}{cc}
-\left(2 \mathbb{E}+l^{2}\right) \tilde{x}_{2} & \left(2 \mathbb{E}+l^{2}\right) l^{2} \\
-2 \mathbb{N} \tilde{x}_{2} & 2 \mathbb{N} l^{2}
\end{array}\right),
\end{aligned}
$$

where $\Delta=\tilde{x}_{2}^{2}+(\beta-1) l^{4}$. Finally, one can write down the action of dual $\sigma$-model in the following form

$$
\begin{aligned}
\tilde{S}= & \frac{1}{2} \int d \sigma^{+} d \sigma^{-}\left\{-l^{2}\left(1-\frac{4 \mathbb{E}^{2}-l^{4}}{\Delta}\right) \partial_{+} t \partial_{-} t\right. \\
& +l^{2}\left(1+\frac{4 \mathbb{N}^{2}}{\Delta}\right) \partial_{+} z \partial_{-} z \\
& +\frac{1}{\Delta}\left[l^{2}(\beta-1) \partial_{+} \tilde{x}_{1} \partial_{-} \tilde{x}_{1}+l^{2} \partial_{+} \tilde{x}_{2} \partial_{-} \tilde{x}_{2}\right. \\
& +\tilde{x}_{2}\left(\partial_{+} \tilde{x}_{1} \partial_{-} \tilde{x}_{2}-\partial_{+} \tilde{x}_{2} \partial_{-} \tilde{x}_{1}\right) \\
& +2 \mathbb{N} \tilde{x}_{2}\left(\partial_{+} \tilde{x}_{1} \partial_{-} z-\partial_{+} z \partial_{-} \tilde{x}_{1}\right) \\
& +2 \mathbb{N} l^{2}\left(\partial_{+} \tilde{x}_{2} \partial_{-} z+\partial_{+} z \partial_{-} \tilde{x}_{2}\right) \\
& +\left(2 \mathbb{E}-l^{2}\right)\left(\tilde{x}_{2} \partial_{+} \tilde{x}_{1} \partial_{-} t\right. \\
& \left.+l^{2} \partial_{+} \tilde{x}_{2} \partial_{-} t+2 \mathbb{N} l^{2} \partial_{+} z \partial_{-} t\right) \\
& +\left(2 \mathbb{E}+l^{2}\right)\left(-\tilde{x}_{2} \partial_{+} t \partial_{-} \tilde{x}_{1}\right. \\
& \left.\left.\left.+l^{2} \partial_{+} t \partial_{-} \tilde{x}_{2}+2 \mathbb{N} l^{2} \partial_{+} t \partial_{-} z\right)\right]\right\} \\
& -\frac{1}{4 \pi} \int d \sigma^{+} d \sigma^{-} R^{(2)} \tilde{\Phi}(\tilde{X}) .
\end{aligned}
$$

Comparing the above action with the $\sigma$-model action of the form (2.1), the line element and the tensor field $\tilde{B}$ take the following forms

$$
\begin{aligned}
d \tilde{s}^{2}= & \frac{1}{\Delta}\left[-l^{2}\left(\tilde{x}_{2}^{2}+\beta l^{4}-4 \mathbb{E}^{2}\right) d t^{2}+l^{2}\left(\tilde{x}_{2}^{2}+(\beta-1) l^{4}\right.\right. \\
& \left.+4 \mathbb{N}^{2}\right) d z^{2}+l^{2}(\beta-1) d \tilde{x}_{1}^{2} \\
& +l^{2} d \tilde{x}_{2}^{2}-2 l^{2} \tilde{x}_{2} d \tilde{x}_{1} d t+4 \mathbb{E} l^{2} d \tilde{x}_{2} d t+4 \mathbb{N} l^{2} d \tilde{x}_{2} d z \\
& \left.+8 \mathbb{E} \mathbb{N} l^{2} d z d t\right], \\
\tilde{B}= & \frac{1}{\Delta}\left[\tilde{x}_{2} d \tilde{x}_{1} \wedge d \tilde{x}_{2}+2 \mathbb{E} \tilde{x}_{2} d \tilde{x}_{1} \wedge d t+2 \mathbb{N} \tilde{x}_{2} d \tilde{x}_{1} \wedge d z\right. \\
& \left.-l^{4} d \tilde{x}_{2} \wedge d t-2 \mathbb{N} l^{4} d z \wedge d t\right] .
\end{aligned}
$$

In order to enhance and clarify the structure of the dual spacetime, we can test whether there are true singularities by calculating the scalar curvature, which is

$\tilde{\mathcal{R}}=\frac{1}{2 \beta l^{2} \Delta^{2}}\left[(1-4 \beta) \tilde{x}_{2}^{4}+\left(4 \mathbb{N}^{2}(1-20 \beta)\right.\right.$

$$
\begin{aligned}
& \left.+2(\beta-1)\left(l^{4}+40 \mathbb{E}^{2}\right)\right) \tilde{x}_{2}^{2} \\
& -32 \mathbb{E}^{2} l^{4}(\beta-1)^{2}+4 \mathbb{N}^{2} l^{4}(\beta-1)(1+8 \beta) \\
& \left.+l^{8}(\beta-1)^{2}(1+4 \beta)\right] .
\end{aligned}
$$

As it is seen from the formulas (7.13) and (7.15), the regions $\tilde{x}_{2}= \pm l^{2} \sqrt{(1-\beta)}$ are true curvature singularities. Therefore, the dual metric has true singularities for the range $0<\beta<1$. The results indicate that the dual spacetime corresponding to the Gödel metrics allowing CTC's has true singularities, because the metric (3.12) allows CTC's for the range $0<\beta<1$ as mentioned in Sect. 3 .

In order to investigate the conformal invariance conditions of the dual model up to the first order in $\alpha^{\prime}$, we look at the two-loop beta-function equations. Before proceeding to this, one easily gets that the field strength corresponding to the $\tilde{B}$-field (7.14) is

$$
\begin{aligned}
\tilde{H}= & \frac{1}{\Delta^{2}}\left[\mathbb{E}\left(\tilde{x}_{2}^{2}-(\beta-1) l^{4}\right) d \tilde{x}_{1} \wedge d \tilde{x}_{2} \wedge d t\right. \\
& +\mathbb{N}\left(\tilde{x}_{2}^{2}-(\beta-1) l^{4}\right) d \tilde{x}_{1} \wedge d \tilde{x}_{2} \wedge d z \\
& \left.-2 \mathbb{N} l^{4} \tilde{x}_{2} d \tilde{x}_{2} \wedge d t \wedge d z\right] .
\end{aligned}
$$

Now by solving the field equations (2.2)-(2.4) for the metric (7.13) and the field strength (7.16) one concludes that there is no suitable dilaton field to satisfy these equations. In the next subsection, we study the non-Abelian T-duality of the Gödel spacetimes by constructing a pair of the mutually dual $\sigma$-models on 3+1-dimensional manifolds with the $A_{2} \oplus A_{1}$ and $3 A_{1}$ Lie groups. It is then shown the T-dual $\sigma$-models that are conformal at one-loop remain conformal at two-loop.

7.2 Non-Abelian T-duality from a $3+1$-dimensional manifold with the $A_{2} \oplus A_{1}$ Lie group

There is a possibility that we can also get the Gödel spacetimes from a T-dualizable $\sigma$-model constructing on a $3+1$ dimensional manifold $\mathcal{M} \approx O \times G$, in which $G$ is threedimensional decomposable Lie group $A_{2} \oplus A_{1}{ }^{5}$ acting freely on $\mathcal{M}$, while the orbit $O$ is, here, a one-dimensional space with time coordinate $y^{i}=\{t\}$. The dual model is constructed on manifold $\tilde{\mathcal{M}} \approx O \times \tilde{G}$, where $\tilde{G}$ is threedimensional Abelian Lie group $3 A_{1}$. The Lie algebra of the semi-Abelian double $\left(\mathcal{A}_{2} \oplus \mathcal{A}_{1}, 3 \mathcal{A}_{1}\right)$ is generated by the generators $\left(T_{a}, \tilde{T}^{a}\right)$ with the following Lie brackets [59-61]

$\left[T_{1}, T_{2}\right]=T_{2}, \quad\left[T_{1}, \tilde{T}^{2}\right]=-\tilde{T}^{2}, \quad\left[T_{2}, \tilde{T}^{2}\right]=\tilde{T}^{1}, \quad\left[T_{3},.\right]=0$, $\left[\tilde{T}^{1},.\right]=0, \quad\left[\tilde{T}^{3},.\right]=0$.

Taking a convenient element of the Lie group $A_{2} \oplus A_{1}$,

$g=e^{x_{1} T_{1}} e^{x_{2} T_{2}} e^{x_{3} T_{3}}$,

\footnotetext{
5 Notice that the Lie algebra $\mathcal{A}_{2} \oplus \mathcal{A}_{1}$ of the Lie group $A_{2} \oplus A_{1}$ is isomorphic to the Lie algebra of Bianchi type III.
} 
where $\left(x_{1}, x_{2}, x_{3}\right)$ stand for the coordinates of $A_{2} \oplus A_{1}$, we immediately find that $R_{ \pm}^{1}=\partial_{ \pm} x_{1}, R_{ \pm}^{2}=e^{x_{1}} \partial_{ \pm} x_{2}$ and $R_{ \pm}^{3}=\partial_{ \pm} x_{3}$. In addition, we parameterize the Lie group $3 A_{1}$ with coordinates $\left(\tilde{x}_{1}, \tilde{x}_{2}, \tilde{x}_{3}\right)$ so that its element is defined as in (7.18) by replacing untilded quantities with tilded ones. Hence, using (4.4) with and without tilded quantities we obtain

$$
\Pi^{a b}(g)=0, \quad \tilde{\Pi}_{a b}(\tilde{g})=\left(\begin{array}{ccc}
0 & -\tilde{x}_{2} & 0 \\
\tilde{x}_{2} & 0 & 0 \\
0 & 0 & 0
\end{array}\right) .
$$

The coupling matrices of the original $\sigma$-model are, here, obtained by considering the spectator-dependent background matrices

$E_{0 a b}=\left(\begin{array}{ccc}l^{2} & s & 0 \\ -s & (\beta-1) l^{2} & 2 \mathbb{N} \\ 0 & -2 \mathbb{N} & l^{2}\end{array}\right), F_{a j}^{(1)}=\left(\begin{array}{c}0 \\ 2 \mathbb{E}-l^{2} \\ 0\end{array}\right)$,

$$
F_{i b}^{(2)}=\left(\begin{array}{lll}
0 & -\left(2 \mathbb{E}+l^{2}\right) & 0
\end{array}\right) \text {, }
$$

and $F_{i j}=-l^{2}$ for some constant $s$. Inserting (7.19) and (7.20) into Eqs. (4.6)-(4.9), and using (4.5), then we obtain the original $\sigma$-model in the following form

$$
\begin{aligned}
S= & \frac{1}{2} \int d \sigma^{+} d \sigma^{-}\left[l^{2} \partial_{+} x_{1} \partial_{-} x_{1}\right. \\
& +l^{2}(\beta-1) e^{2 x_{1}} \partial_{+} x_{2} \partial_{-} x_{2}+\left(2 \mathbb{E}-l^{2}\right) e^{x_{1}} \partial_{+} x_{2} \partial_{-} t \\
& -\left(2 \mathbb{E}+l^{2}\right) e^{x_{1}} \partial_{+} t \partial_{-} x_{2}+2 \mathbb{N} e^{x_{1}}\left(\partial_{+} x_{2} \partial_{-} x_{3}-\partial_{+} x_{3} \partial_{-} x_{2}\right) \\
& \left.-l^{2} \partial_{+} t \partial_{-} t+l^{2} \partial_{+} x_{3} \partial_{-} x_{3}+s e^{x_{1}}\left(\partial_{+} x_{1} \partial_{-} x_{2}-\partial_{+} x_{2} \partial_{-} x_{1}\right)\right] \\
& -\frac{1}{4 \pi} \int d \sigma^{+} d \sigma^{-} R^{(2)} \Phi(X) .
\end{aligned}
$$

Utilizing the coordinate transformation $e^{x_{1}}=r, x_{2}=$ $\varphi, x_{3}=z$, the metric of the model becomes the same as the Gödel metric (3.12), and $B$-field is given by

$B=2 \mathbb{E} r d \varphi \wedge d t+2 \mathbb{N} r d \varphi \wedge d z+s d r \wedge d \varphi$

such that the corresponding field strength takes the same form as (3.22). One can check that only the case of $\beta=1$ of the model (7.21) with conditions $\mathbb{E}^{2}=l^{4} / 4$ and $\mathbb{N}=0$ is conformally invariant up to zeroth order in $\alpha^{\prime}$. The dilaton filed that makes the model conformal is obtained by using equation (4.15) to be $\Phi=\phi^{(0)}+x_{1}=\phi^{(0)}+\log r$ such that $\phi^{(0)}$ must be $\phi^{(0)}=f z-\log r+b$. Finally we get

$\Phi=f z+b$,

where $f^{2}=-1+2 l^{2} \Lambda$.

In order to obtain the dual $\sigma$-model for (7.21), we use the action (4.10). The dual coupling matrices can be obtained by inserting (7.19) and (7.20) into (4.11)-(4.14). Finally, the dual background is read

$$
\begin{aligned}
d \tilde{s}^{2}= & \frac{1}{\hat{\Delta}}\left\{-l^{2}\left[\left(s-\tilde{x}_{2}\right)^{2}+\beta l^{4}+4\left(\mathbb{N}^{2}-\mathbb{E}^{2}\right)\right] d t^{2}\right. \\
& +\frac{1}{l^{2}}\left[4 \mathbb{N}^{2}+l^{4}(\beta-1)\right] d \tilde{x}_{1}^{2}+l^{2} d \tilde{x}_{2}^{2}+\frac{1}{l^{2}}\left[\left(s-\tilde{x}_{2}\right)^{2}\right. \\
& \left.+l^{4}(\beta-1)\right] d \tilde{x}_{3}^{2}-4 \mathbb{N} l^{2} d \tilde{x}_{3} d t+4 \mathbb{E} l^{2} d \tilde{x}_{2} d t \\
& \left.+2 l^{2}\left(s-\tilde{x}_{2}\right) d \tilde{x}_{1} d t+\frac{4}{l^{2}} \mathbb{N}\left(s-\tilde{x}_{2}\right) d \tilde{x}_{1} d \tilde{x}_{3}\right\}, \\
\tilde{B}= & \frac{1}{\hat{\Delta}}\left\{\left(\tilde{x}_{2}-s\right) d \tilde{x}_{1} \wedge d \tilde{x}_{2}+2 \mathbb{E}\left(\tilde{x}_{2}-s\right) d \tilde{x}_{1} \wedge d t\right. \\
& \left.-2 \mathbb{N} d \tilde{x}_{2} \wedge d \tilde{x}_{3}-l^{4} d \tilde{x}_{2} \wedge d t+4 \mathbb{E} \mathbb{N} d \tilde{x}_{3} \wedge d t\right\},
\end{aligned}
$$

where $\hat{\Delta}=\left(s-\tilde{x}_{2}\right)^{2}+4 \mathbb{N}^{2}+(\beta-1) l^{4}$. One immediately gets that the only nonzero components of the field strength corresponding to the $\tilde{B}$-field (7.25) are

$$
\begin{aligned}
\tilde{H}_{\tilde{x}_{1} \tilde{x}_{2} t} & =\frac{\mathbb{E}}{\hat{\Delta}^{2}}\left[\left(s-\tilde{x}_{2}\right)^{2}-4 \mathbb{N}^{2}-(\beta-1) l^{4}\right], \\
\tilde{H}_{\tilde{x}_{2} \tilde{x}_{3} t} & =\frac{4 \mathbb{E} \mathbb{N}\left(s-\tilde{x}_{2}\right)}{\hat{\Delta}^{2}} .
\end{aligned}
$$

Before proceeding to investigate the conformal invariance conditions of the dual background, let us enhance and clarify the structure of the dual spacetime. Similar to the original model we impose the conditions $\beta=1, \mathbb{E}^{2}=l^{4} / 4$ and $\mathbb{N}=0$ on the dual solution (Eqs. (7.24) and (7.25)), then by shifting $\tilde{x}_{2}$ to $\tilde{x}_{2}+s$ we get

$$
\begin{aligned}
d \tilde{s}^{2} & =-l^{2} d t^{2}+\frac{1}{l^{2}} d \tilde{x}_{3}^{2}+\frac{l^{2}}{\tilde{x}_{2}^{2}} d \tilde{x}_{2}^{2}-\frac{2 l^{2}}{\tilde{x}_{2}}\left(d \tilde{x}_{1} d t-\frac{l^{2}}{\tilde{x}_{2}} d \tilde{x}_{2} d t\right), \\
\tilde{B} & =\frac{1}{\tilde{x}_{2}}\left[d \tilde{x}_{1} \wedge d \tilde{x}_{2}+l^{2} d \tilde{x}_{1} \wedge d t-\frac{l^{4}}{\tilde{x}_{2}} d \tilde{x}_{2} \wedge d t\right] .
\end{aligned}
$$

Here we have used $\mathbb{E}=l^{2} / 2$. To have a better understanding of the structure of metric (7.27), we use the coordinate transformation

$\tilde{x}_{1}=-l^{2} \ln r+\varphi, \quad \tilde{x}_{2}=\frac{1}{r}, \quad \tilde{x}_{3}=l^{2} z$.

Accordingly, the metric (7.27) turns into the case of $\beta=1$ of the Gödel metric (3.12). ${ }^{6}$ This particular case of the dual solution is conformally invariant up to the one-loop order with a new dilaton field which is obtained by using Eqs. (4.16) and (7.23), giving

$\tilde{\Phi}=\tilde{f} \tilde{x}_{3}+\tilde{b}=\tilde{f} l^{2} z+\tilde{b}$.

where $\tilde{f}^{2}=\left(2 l^{2} \Lambda-1\right) / l^{4}$. It should be noted that the dual background given by Eqs. (7.27) and (7.28) is also conformally invariant up to two-loop order with the same dilaton

\footnotetext{
${ }^{6}$ Notice that the Gödel metric (3.12) with the condition $\beta=1$ represents the $A d S_{3} \times \mathbb{R}$ space locally as shown in Eq. (3.15).
} 
filed (7.30). After performing the transformation (7.29) on the $\tilde{B}$-field (7.28), the only nonzero component of the corresponding field strength becomes $\tilde{H}_{t r \varphi}=l^{2} / 2$. This result is in agreement with the case $\left(1^{\prime}\right)$ of the solutions of class A. Thus, we showed that the $A d S_{3} \times \mathbb{R}$ space does remain invariant under the non-Abelian T-duality transformation, that is, the model is PL self-dual. If one uses the conditions $\beta=1, \mathbb{N}=0$ and $\mathbb{E}=-l^{2} / 2$, then, they will obtain similar results.

\subsection{Non-Abelian T-duality from a $3+1$-dimensional manifold with the $S L(2, \mathbb{R})$ Lie group}

In the preceding subsection, we studied the non-Abelian T-duality of the Gödel spacetimes by applying the $3+1$ dimensional manifold $\mathcal{M} \approx O \times G$ with $G=A_{2} \oplus A_{1}$. In addition, we can only derive the case of $\beta=1$ of the Gödel metrics from a $3+1$-dimensional manifold with the $S L(2, \mathbb{R})$ Lie group and then obtain the corresponding dual spacetime. In this way, the pair of the mutually dual models are constructed on semi-Abelian double $\left(\operatorname{sl}(2, \mathbb{R}), 3 \mathcal{A}_{1}\right)$. The Lie algebra of double $\left(\operatorname{sl}(2, \mathbb{R}), 3 \mathcal{A}_{1}\right)$ is defined by nonzero Lie brackets as [59-61],

$\left[T_{1}, T_{2}\right]=T_{2}, \quad\left[T_{1}, T_{3}\right]=-T_{3}, \quad\left[T_{2}, T_{3}\right]=2 T_{1}, \quad\left[T_{1}, \tilde{T}^{2}\right]=-\tilde{T}^{2}$, $\left[T_{1}, \tilde{T}^{3}\right]=\tilde{T}^{3},\left[T_{2}, \tilde{T}^{1}\right]=-2 \tilde{T}^{3},\left[T_{2}, \tilde{T}^{2}\right]=\tilde{T}^{1},\left[T_{3}, \tilde{T}^{3}\right]=-\tilde{T}^{1}$, $\left[T_{3}, \tilde{T}^{1}\right]=-2 \tilde{T}^{2}$.

where $\left(T_{1}, T_{2}, T_{3}\right)$ and $\left(\tilde{T}^{1}, \tilde{T}^{2}, \tilde{T}^{3}\right)$ are the basis of the Lie algebras $s l(2, \mathbb{R})$ and $3 \mathcal{A}_{1}$, respectively. We note that the double $\left(s l(2, \mathbb{R}), 3 \mathcal{A}_{1}\right)$ has the vanishing trace in the adjoint representations. In such a situation, at the one-loop level a conformally invariant $\sigma$-model leads, under the PL duality, to a dual theory with the same property [55]. Now we parametrize an element of $S L(2, \mathbb{R})$ as

$g=e^{x_{2} T_{2}} e^{x_{1} T_{1}} e^{x_{3} T_{3}}$,

where $\left(x_{1}, x_{2}, x_{3}\right)$ are the coordinates of $S L(2, \mathbb{R})$. Then one gets the corresponding one-forms components in the following way

$R_{ \pm}^{1}=\partial_{ \pm} x_{1}+2 e^{-x_{1}} x_{2} \partial_{ \pm} x_{3}$,

$R_{ \pm}^{2}=-x_{2} \partial_{ \pm} x_{1}+\partial_{ \pm} x_{2}-x_{2}^{2} e^{-x_{1}} \partial_{ \pm} x_{3}$,

$R_{ \pm}^{3}=e^{-x_{1}} \partial_{ \pm} x_{3}$.

Since the dual Lie group is considered to be Abelian, hence, by using (4.4) and (7.31) it is followed that $\Pi(g)=0$. Using the above results and also choosing the spectator-dependent matrices in the form

$$
E_{0 a b}=\left(\begin{array}{ccc}
\frac{l^{2}}{4} & 0 & 0 \\
0 & 0 & \frac{l^{2}}{2}+\mathbb{E} \\
0 & \frac{l^{2}}{2}-\mathbb{E} & 0
\end{array}\right),
$$

$$
F_{a j}^{(1)}=0, \quad F_{i b}^{(2)}=0, \quad F_{i j}=l^{2},
$$

we obtain the background of the original $\sigma$-model. It is given by

$$
\begin{aligned}
d s^{2} & =l^{2}\left(\frac{1}{4} d x_{1}^{2}+e^{-x_{1}} d x_{2} d x_{3}+d z^{2}\right), \\
B & =\mathbb{E} e^{-x_{1}}\left(-x_{2} d x_{1} \wedge d x_{3}+d x_{2} \wedge d x_{3}\right),
\end{aligned}
$$

where $z$ stands for the coordinate of the orbit $O$ of $G$. The metric (7.35) can be written as $d s^{2}=d s_{A d S_{3}}^{2}+l^{2} d z^{2}$. In order to get more insight of this metric one may use the following transformation

$e^{\frac{x_{1}}{2}}=\frac{1}{\rho}, \quad x_{2}=\frac{1}{l}(\tau-x), \quad x_{3}=-l(\tau+x)$,

then, the metric becomes the same as the $A d S_{3} \times \mathbb{R}$ space given by (3.15). As explained in Sect. 3, the metric (3.15) is locally equivalent to the case of $\beta=1$ of the Gödel metric (3.12). In addition, one easily gets that the field strength corresponding to the $B$-field (7.36) is zero. We have checked that the metric constructed on the double $\left(\operatorname{sl}(2, \mathbb{R}), 3 \mathcal{A}_{1}\right)$ with a vanishing field strength can't be conformally invariant up to the one-loop order. Therefore, according to Ref. [55] we don't expect to have a conformally invariant dual theory at one-loop level. One immediately verifies the field equations (2.2)-(2.4) for the metric (7.35) ${ }^{7}$ and vanishing field strength together with the dilaton field $\Phi=f z+b$ for which $f^{2}=2 l^{2} \Lambda-3$; moreover, to satisfy the field equations we must have a coupling constant in the form of $\alpha^{\prime}=l^{2}$.

In the same way, to construct out the dual $\sigma$-model on the manifold $\tilde{\mathcal{M}} \approx O \times \tilde{G}$ with the Lie group $3 A_{1}$ we parameterize the corresponding Lie group with coordinates $\left(\tilde{x}_{1}, \tilde{x}_{2}, \tilde{x}_{3}\right)$ so that its element is defined as (7.32) by replacing untilded quantities with tilded ones. Utilizing the relation (7.31) and also (4.4) for tilded quantities we get

$\tilde{\Pi}_{a b}(\tilde{g})=\left(\begin{array}{ccc}0 & -\tilde{x}_{2} & \tilde{x}_{3} \\ \tilde{x}_{2} & 0 & -2 \tilde{x}_{1} \\ -\tilde{x}_{3} & 2 \tilde{x}_{1} & 0\end{array}\right)$.

Finally, by using (4.10) the metric and $\tilde{B}$-field of the dual model take the following forms

$$
\begin{aligned}
d \tilde{s}^{2}= & l^{2} d z^{2}+\frac{4}{\tilde{\Delta}}\left[l ^ { 2 } \left(d \tilde{x}_{1}^{\prime 2}\right.\right. \\
& \left.\left.+d \tilde{x}_{2} d \tilde{x}_{3}\right)-\frac{4}{l^{2}}\left(2 \tilde{x}_{1}^{\prime} d \tilde{x}_{1}^{\prime}+d\left(\tilde{x}_{2} \tilde{x}_{3}\right)\right)^{2}\right],
\end{aligned}
$$

\footnotetext{
${ }^{7}$ Considering $z$ as the time coordinate in (7.35) one obtains an anisotropic homogeneous spacetime based on the $S L(2, \mathbb{R})$ Lie group so that it is a solution for two-loop beta-function equations. Recently, the two-loop beta-function equations with dilaton and $B$-field on the anisotropic homogeneous spacetimes of the Bianchi-type have been investigated [62].
} 


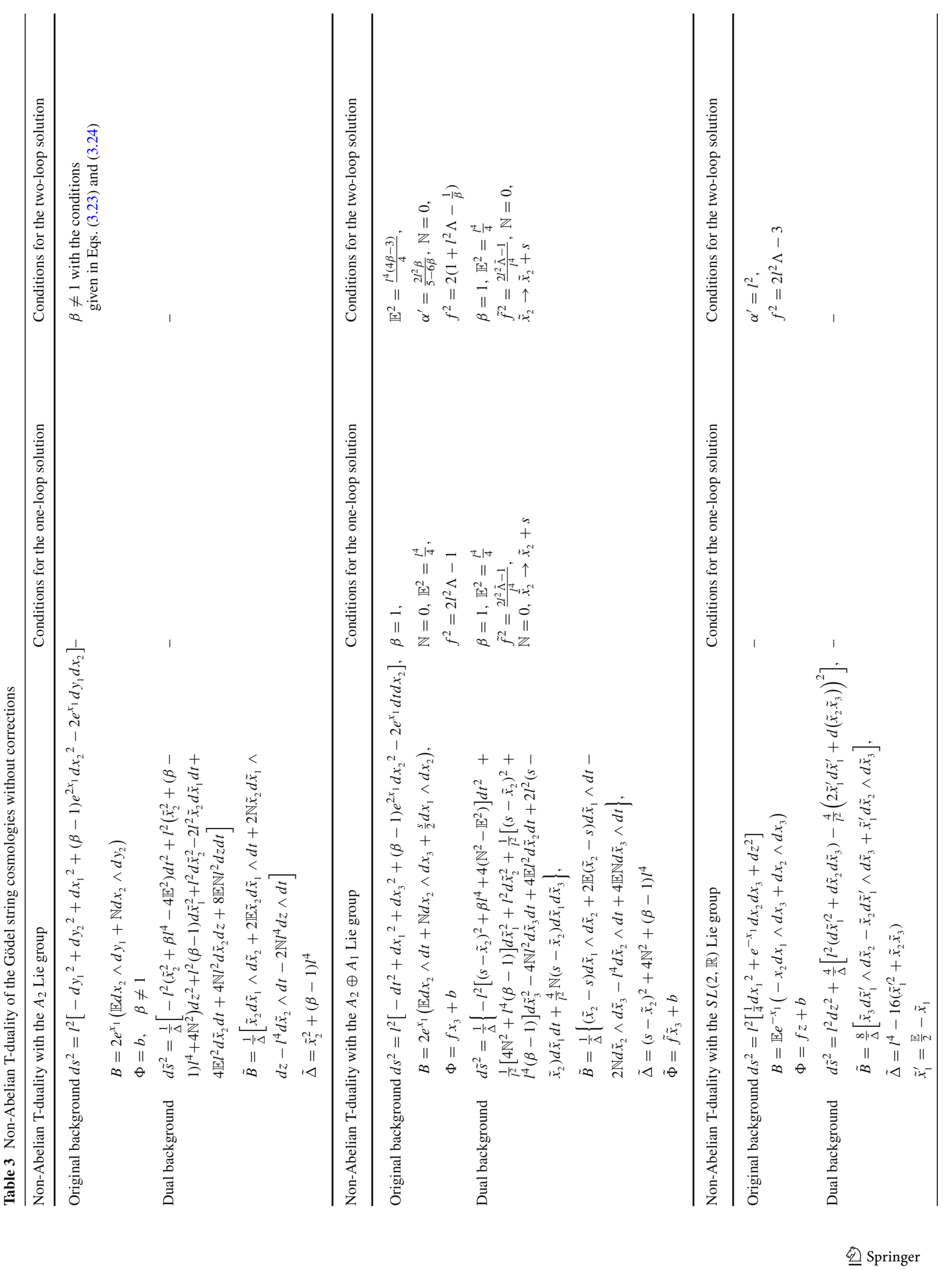




$$
\tilde{B}=\frac{8}{\tilde{\Delta}}\left[\tilde{x}_{3} d \tilde{x}_{1}^{\prime} \wedge d \tilde{x}_{2}-\tilde{x}_{2} d \tilde{x}_{1}^{\prime} \wedge d \tilde{x}_{3}+\tilde{x}_{1}^{\prime} d \tilde{x}_{2} \wedge d \tilde{x}_{3}\right]
$$

where $\tilde{\Delta}=l^{4}-16\left(\tilde{x}_{1}^{\prime 2}+\tilde{x}_{2} \tilde{x}_{3}\right)$ and $\tilde{x}_{1}^{\prime}=\mathbb{E} / 2-\tilde{x}_{1}$. For the $\tilde{B}$-field there is only a nonzero component of the field strength, obtaining

$$
\tilde{H}_{\tilde{x}_{1}^{\prime} \tilde{x}_{2} \tilde{x}_{3}}=\frac{4}{\tilde{\Delta}^{2}}\left[3 l^{4}-16\left(\tilde{x}_{1}^{\prime 2}+\tilde{x}_{2} \tilde{x}_{3}\right)\right] .
$$

As mentioned above, since the background of the original $\sigma$ model doesn't satisfy the one-loop beta-function equations, the dual background can't be conformally invariant at the one-loop order. We have represented the results of the nonAbelian T-duality of the Gödel string cosmologies in Table 3.

\section{Conclusion}

We have obtained some new solutions for the field equations of bosonic string effective action up to first order in $\alpha^{\prime}$, including the Gödel spacetimes, axion field and dilaton. Our results have shown that these solutions can be appropriate to study (non-)Abelian T-dualization of Gödel string cosmologies via PL T-duality approach. In studying Abelian duality of the Gödel spacetimes we have found seven dual models in a way that the models are constructed by one-, two- and three-dimensional Abelian Lie groups acting freely on the target space manifold. When the dualizing was implemented by the shift of directions $t, z$ and $(t, z)$ we showed that the pair of the mutually dual models can be conformally invariant at one-loop level, in a way that the corresponding dual dilaton field was found by using transformation (4.17). Our results showed that the Abelian T-dual models are, under some of the special conditions, self-dual; moreover, by applying the rules of Abelian T-duality without further corrections, we were still able to obtain two-loop solutions. We also studied the Abelian T-duality of Gödel string cosmologies up to $\alpha^{\prime}$-corrections by using the T-duality rules at two-loop order of KM. Most importantly, we have obtained the nonAbelian duals of the Gödel spacetimes. First, we have constructed the T-dual models on the four-dimensional manifold $\mathcal{M} \approx O \times G$ with two-dimensional non-Abelian Lie group and have shown that the metric of the dual model has true singularities for the range of $0<\beta<1$. In this case, the models are valid for all values of $\beta$ except for 1 . Unfortunately, the dual model doesn't satisfy the two-loop beta-function equations. We have then found other non-Abelian duals for the Gödel spacetimes by applying the $A_{2} \oplus A_{1}$ and $S L(2, \mathbb{R})$ Lie groups. The case of $\beta=1$ of dual model constructed on the semi-Abelian double $\left(\mathcal{A}_{2} \oplus \mathcal{A}_{1}, 3 \mathcal{A}_{1}\right)$ is conformally invariant to zeroth order in $\alpha^{\prime}$, as well as to the first order.
In this way, it has shown that the $\beta=1$ case of the pair of the mutually dual models as the $\operatorname{AdS}_{3} \times \mathbb{R}$ spaces are PL self-dual. Finally, we have shown that the model constructed by the double $\left(\operatorname{sl}(2, \mathbb{R}), 3 \mathcal{A}_{1}\right)$ leads to the case of $\beta=1$ of the Gödel metrics with zero field strength. Indeed, the model didn't satisfy the one-loop beta-function equations. Because of the vanishing traces of the structure constants corresponding to the double $\left(\operatorname{sl}(2, \mathbb{R}), 3 \mathcal{A}_{1}\right)$, the dual model couldn't be also conformally invariant at the one-loop order.

Acknowledgements This work has been supported by the research vice chancellor of Azarbaijan Shahid Madani University under research fund No. 97/231. A. Eghbali is especially grateful to A. Mehrvand for his careful reading of the manuscript.

Data Availability Statement This manuscript has no associated data or the data will not be deposited. [Authors' comment: There are no date deposited since this is a theoretical work.].

Open Access This article is licensed under a Creative Commons Attribution 4.0 International License, which permits use, sharing, adaptation, distribution and reproduction in any medium or format, as long as you give appropriate credit to the original author(s) and the source, provide a link to the Creative Commons licence, and indicate if changes were made. The images or other third party material in this article are included in the article's Creative Commons licence, unless indicated otherwise in a credit line to the material. If material is not included in the article's Creative Commons licence and your intended use is not permitted by statutory regulation or exceeds the permitted use, you will need to obtain permission directly from the copyright holder. To view a copy of this licence, visit http://creativecomm ons.org/licenses/by/4.0/.

Funded by SCOAP ${ }^{3}$.

\section{References}

1. K. Gödel, An example of a new type of cosmological solutions of Einstein's field equations of gravitation. Rev. Mod. Phys. 21, 447 (1949)

2. J. D. Barrow, M. P. Dąbrowski, Gödel universes in string theory. Phys. Rev. D 58, 103502 (1998)

3. S. L. Li, X. H. Feng, H. Wei, H. Lu, Gödel universe from string theory. Eur. Phys. J. C 77, 289 (2017). arXiv:1612.02069 [hep-th]

4. Dan Israël, Quantization of heterotic strings in a Gödel/anti de Sitter spacetime and chronology protection. J. High Energy Phys. 01, 042 (2004). arXiv:hep-th/0310158

5. T. Buscher, A symmetry of the string background field equations. Phys. Lett. B 194, 59 (1987)

6. T. Buscher, Path-integral derivation of quantum duality in nonlinear sigma-models. Phys. Lett. B 201, 466 (1988)

7. A. Giveon, M. Porrati, E. Rabinovici, Target space duality in string theory. Phys. Rep. 244, 77 (1994)

8. X. C. de la Ossa, F. Quevedo, Duality symmetries from non-abelian isometries in string theory. Nucl. Phys. B 403, 377 (1993)

9. M. Rocek, E. Verlinde, Duality, quotients, and currents. Nucl. Phys. B 373, 630 (1992)

10. M. Gasperini, R. Ricci, G. Veneziano, A problem with non-abelian duality? Phys. Lett. B 319, 438 (1993)

11. A. Givoen, M. Roček, On nonabelian duality. Nucl. Phys. B 421, 173 (1994) 
12. E. Alvarez, A. Alvarez-Gaume, Y. Lozano, On non-abelian duality. Nucl. Phys. B 424, 155 (1994)

13. E. Tyurin, On conformal properties of the dualized sigma-models. Phys. Lett. B 348, 386 (1995)

14. S. Elitzur, A. Giveon, E. Rabinovici, A. Schwimmer, G. Veneziano, Remarks on non-Abelian duality. Nucl. Phys. B 435, 147 (1995)

15. K. Sfetsos, D. C. Thompson, On non-abelian T-dual geometries with Ramond fluxes. Nucl. Phys. B 846, 21 (2011). arXiv:1012.1320 [hep-th]

16. Y. Lozano, E. Ó. Colgáin, K. Sfetsos, D. C. Thompson, Non-abelian T-duality, Ramond fields and coset geometries. J. High Energy Phys. 06, 106 (2011). arXiv:1104.5196 [hep-th]

17. G. Itsios, C. Núñez, K. Sfetsos, D. C. Thompson, Non-Abelian T-duality and the AdS/CFT correspondence: new $N=1$ backgrounds. Nucl. Phys. B 873, 1 (2013). arXiv:1301.6755 [hep-th]

18. K. Sfetsos, Integrable interpolations: from exact CFTs to nonAbelian T-duals. Nucl. Phys. B 880, 225 (2014). arXiv:1312.4560 [hep-th]

19. G. Itsios, Y. Lozano, J. Montero, C. Núñez, The $A d S_{5}$ non-Abelian T-dual of Klebanov-Witten as a $\mathcal{N}=1$ linear quiver from $M_{5^{-}}$ branes. J. High Energy Phys. 09, 038 (2017). arXiv:1705.09661 [hep-th]

20. G. Itsios, H. Nastase, C. Núñez, K. Sfetsos, S. Zacarías, Penrose limits of Abelian and non-Abelian T-duals of $A d S_{5} \times S^{5}$ and their field theory duals. J. High Energy Phys. 01, 071 (2018). arXiv:1711.09911 [hep-th]

21. C. Klimčík, P. Ševera, Dual non-Abelian duality and the Drinfeld double. Phys. Lett. B 351, 455 (1995). arXiv:hep-th/9502122

22. C. Klimčík, Poisson-Lie T-duality. Nucl. Phys. Proc. Suppl. B 46, 116 (1996). arXiv:hep-th/9509095

23. A. Eghbali, A. Rezaei-Aghdam, Poisson-Lie T-dual sigma models on supermanifolds. J. High Energy Phys. 09, 094 (2009). arXiv:0901.1592 [hep-th]

24. A. Eghbali, A. Rezaei-Aghdam, String cosmology from PoissonLie T-dual sigma models on supermanifolds. J. High Energy Phys. 01, 151 (2012). arXiv:1107.2041 [hep-th]

25. A. Eghbali, A. Rezaei-Aghdam, Super Poisson-Lie symmetry of the $G L(1 \mid 1)$ WZNW model and worldsheet boundary conditions. Nucl. Phys. B 866, 26 (2013). arXiv:1207.2304 [hep-th]

26. A. Eghbali, A. Rezaei-Aghdam, WZW models as mutual super Poisson-Lie T-dual sigma models. J. High Energy Phys. 07, 134 (2013). arXiv:1303.4069 [hep-th]

27. A. Alekseev, C. Klimčík, A. Tseytlin, Quantum Poisson-Lie Tduality and WZNW model. Nucl. Phys. B 458, 430 (1996). arXiv:hep-th/9509123

28. K. Sfetsos, Poisson-Lie T-duality beyond the classical level and the renormalization group. Phys. Lett. B 432, 365 (1998). arXiv:hep-th/9803019

29. M. A. Lledo, V. S. Varadarajan, $S U$ (2) Poisson-Lie T duality. Lett. Math. Phys. 45, 247 (1998). arXiv:hep-th/9803175

30. A. Eghbali, A. Rezaei-Aghdam, Poisson Lie symmetry and Dbranes in WZW model on the Heisenberg Lie group $H_{4}$. Nucl. Phys. B 899, 165 (2015). arXiv:1506.06233 [hep-th]

31. R. Borsato, L. Wulff, Integrable deformations of T-dual $\sigma$-models. Phys. Rev. Lett. 117, 251602 (2016). arXiv:1609.09834 [hep-th]

32. R. Borsato, L. Wulff, On non-abelian T-duality and deformations of supercoset string sigma-models. J. High Energy Phys. 10, 024 (2017). arXiv:1706.10169 [hep-th]

33. B. Hoare, F. K. Seibold, Poisson-Lie duals of the $\eta$ deformed symmetric space sigma model. J. High Energy Phys. 11, 014 (2017). arXiv:1709.01448 [hep-th]

34. C. Klimčík, $\eta$ and $\lambda$ deformations as $\mathcal{E}$-models. Nucl. Phys. B 900, 259 (2015). arXiv:1508.05832 [hep-th]

35 . B. Vicedo, Deformed integrable $\sigma$-models, classical $R$-matrices and classical exchange algebra on Drinfel'd doubles. J. Phys. A 48, 355203 (2015). arXiv:1504.06303 [hep-th]
36. B. Hoare, A. A. Tseytlin, On integrable deformations of superstring sigma models related to $A d S_{n} \times S^{n}$ supercosets. Nucl. Phys. B 897, 448 (2015). arXiv: 1504.07213 [hep-th]

37. K. Sfetsos, K. Siampos, D. C. Thompson, Generalised integrable $\lambda$ - and $\eta$ - deformations and their relation. Nucl. Phys. B 899, 489 (2015). arXiv:1506.05784 [hepth]

38. A. Eghbali, L. Mehran-nia, A. Rezaei-Aghdam, BTZ black hole from Poisson-Lie T-dualizable sigma models with spectators. Phys. Lett. B 772, 791 (2017). arXiv:1705.00458 [hep-th]

39. Ali Eghbali, Exact conformal field theories from mutually T-dualizable $\sigma$-models. Phys. Rev. D 99, 026001 (2019). arXiv:1812.07664 [hep-th]

40. R. Borsato, L. Wulff, Quantum correction to generalized Tdualities. Phys. Rev. Lett. 125, 201603 (2020). arXiv:2007.07902 [hep-th]

41. F. Hassler, T. Rochais, $\alpha^{\prime}$-corrected Poisson-Lie T-duality. Fortschr. Phys. 2000063 (2020). arXiv:2007.07897 [hep-th]

42. T. Codina, D. Marques, Generalized dualities and higher derivatives. J. High Energy Phys. 10, 002 (2020). arXiv:2007.09494 [hepth]

43. N. Kaloper, K. A. Meissner, Duality beyond the first loop. Phys. Rev. D 56, 7940 (1997). arXiv:hep-th/9705193

44. C. G. Callan, D. Friedan, E. Martinec, M. J. Perry, String in background fields. Nucl. Phys. B 262, 593 (1985)

45. A. Sen, Heterotic string in an arbitrary background field. Phys. Rev. D 32, 2102 (1985)

46. A. Sen, Equations of motion for the heterotic string theory from the conformal invariance of the sigma model. Phys. Rev. Lett. 55, 1846 (1985)

47. A. Tseytlin, Conformal anomaly in a two-dimensional sigma model on a curved background and strings. Phys. Lett. B 178, 34 (1986)

48. C. M. Hull, K. Townsend, String effective actions from sigmamodel conformal anomalies. Nucl. Phys. B 301, 197 (1988)

49. R. Metsaev, A. Tseytlin, Order $\alpha^{\prime}$ (two-loop) equivalence of the string equations of motion and the $\sigma$-model Weyl invariance conditions: Dependence on the dilaton and the antisymmetric tensor. Nucl. Phys. B 293, 385 (1987)

50. M. J. Rebouças, M. Novello, The stability of a rotating universe. Astrophys. J. 225, 719 (1978)

51. V. G. Drinfeld, Quantum groups, in Proc. Intern. Cong. Math., Berkeley (1986) vol. 1, (Amer. Math. Soc., 1987), p. 798

52. R. von Unge, Poisson-Lie T-plurality. J. High Energy Phys. 07, 014 (2002). arXiv:hep-th/0205245

53. E. Tyurin, R. von Unge, Poisson-Lie T-duality: the path-integral derivation. Phys. Lett. B 382, 233 (1996). arXiv:hep-th/9512025

54. K. Sfetsos, Canonical equivalence of non-isometric $\sigma$-models and Poisson-Lie T-duality. Nucl. Phys. B 517, 549 (1998). arXiv:hep-th/9710163

55. A. Bossard, N. Mohammedi, Poisson-Lie duality in the string effective action. Nucl. Phys. B 619, 128 (2001). arXiv:hep-th/0106211

56. C. Klimčík, P. Ševera, Poisson-Lie T-duality and loop groups of Drinfeld doubles. Phys. Lett. B 372, 65 (1996). arXiv:hep-th/9512040

57. R. Borsato, L. Wulff, Two-loop conformal invariance for YangBaxter deformed strings. J. High Energy Phys. 03, 126 (2020). arXiv:1910.02011 [hep-th]

58. C. M. Hull, Timelike T-Duality, de Sitter space, large N gauge theories and topological field theory. J. High Energy Phys. 07, 021 (1998). arXiv:hep-th/9806146

59. M. A. Jafarizadeh, A. Rezaei-Aghdam, Poisson-Lie T-duality and Bianchi type algebras. Phys. Lett. B 458, 477 (1999). arXiv:hep-th/9903152

60. L. Snobl, L. Hlavaty, Classification of 6-dimensional real Drinfeld doubles. Int. J. Mod. Phys. A 17, 4043 (2002). arXiv:math.QA/0202210 
61. A. Rezaei-Aghdam, M. Hemmati, A. R. Rastkar, Classification of real three-dimensional Lie bialgebras and their Poisson-Lie groups. J. Phys. A Math. Gen. 38, 3981 (2005). arXiv:math-ph/0412092
62. F. Naderi, A. Rezaei-Aghdam, Anisotropic homogeneous string cosmology with two-loop corrections. Nucl. Phys. B 923, 416 (2017). arXiv:1612.06074 [hep-th] 